\title{
Structural modelling of deformable screens for large door openings
}
Cameron L. Hall ${ }^{1}$
Matthew S. Mason ${ }^{2}$
Steven Psaltis ${ }^{3}$
Matthew Chan ${ }^{4}$
Eamon Conway ${ }^{5}$
Sayyed R. Mirnaziry ${ }^{7}$
Danya Rose ${ }^{8}$
Brody Foy ${ }^{6}$
Stephen Taylor ${ }^{9}$
Jakub Tomczyk ${ }^{10}$

(Received 23 October 2015; revised 27 July 2016)

\begin{abstract}
The mathematical modelling of deformable, permeable screen doors was explored to assess their behaviour under wind loading. A loadresponse model was proposed whereby the wind load was modelled using a simplified approach that allowed it to be approximated as a uniformly distributed pressure load with empirical modification factors applied to relate it to the real case of a door on a building. Several approaches were adopted to model the mechanical behaviour of the door system in response to load, including discrete models based on massspring systems, continuum models based on the membrane equations
\end{abstract}

DOI:10.21914/anziamj.v57i0.10156 gives this article, (c) Austral. Mathematical Soc. 2016. Published August 4, 2016, as part of the Proceedings of the 2015 Mathematics and Statistics in Industry Study Group. ISSN 1445-8810. (Print two pages per sheet of paper.) Copies of this article must not be made otherwise available on the internet; instead link directly to the DOI for this article. 
(including tension modulation in some cases), and computational models using finite element packages. The primary aim of the work was to determine the distribution of wind load to the door's supporting 'tabs' and estimate a failure wind speed. The mass-spring model and the membrane models without tension modulation both generated unrealistic deflection magnitudes in response to wind load, but could be calibrated in future work, and then used to obtain an estimate of the total force on the tabs. A tension-modulated version of the membrane model performed better with regards to deflected shape and magnitude, but time constraints meant that the load on the tabs was not calculated. Preliminary validation experiments were undertaken and deflected shape and magnitude were successfully measured in response to given wind speeds.

\section{Contents}

1 Introduction

2 Background

M59

3 Determining the wind load on a screen door

M62

4 Mass-spring models for the deformation of a screen

M67 4.1 Developing a mass-spring model for the deformation of a door 4.2 Force balance equations for the mass-spring model . . . . . M67 4.3 Results from the mass-spring model . . . . . . . . . . . M71

5 Continuum models for the deformation of a screen M77 5.1 General background to membrane models . . . . . . . . . M77

5.2 Membrane models without tension modulation . . . . . . . M81

5.3 Membrane models with tension modulation . . . . . . . . M90

5.3.1 Tension modulation in a strand . . . . . . . . . M90

5.3.2 Critical loads for an untabbed screen . . . . . . . M92 
5.3.3 Continuum finite element model with tension modula-

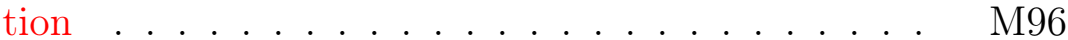

5.4 Membrane models with geometric nonlinearity . . . . . . M99

6 Finite element models of a fibrous screen

M100

7 Validation Experiment

M103

8 Discussion

M106

9 Conclusions

M109

References

M111

\section{Introduction}

Centor is an Australian company that designs and manufactures a range of products for the architectural industry, including folding doors and retractable screens. While Centor originally focused solely on manufacturing hardware for sliding doors, it now designs, manufactures and builds entire door systems and distributes them globally. The products of interest for the 2015 Centor Mathematics-in-Industry Study Group (MISG) are their very large rectractable screens (up to $3 \mathrm{~m}$ by $5 \mathrm{~m}$ ), which roll on to a spool when not in use.

The screen and spools are precision-engineered so that, despite their significant size, the operating friction is sufficiently low that they roll and unroll at the touch of a finger. However, under wind loading screens have been observed to bow substantially, which is aesthetically unappealing. In severe cases this wind load leads to failure of the screen. Centor, through the MISG, are seeking a mathematical tool that will allow them to estimate the size of deflection of their screens under wind loading and to estimate the wind speeds that may lead to failure of the screens. The information will help Centor to 
inform architects and home owners about the appropriate use of the screens to prolong their life, and may be used to guide future design modifications.

At MISG 2015, this project was addressed by dividing it into two parts. One part of the project involved determining the load (expressed as a pressure distribution) that is associated with a particular wind speed. The second part of the project involved determining the screen deflection associated with this pressure distribution, and subsequently the amount of load transferred to its supports. Several mathematical models were implemented to determine these quantities. Considering these data against a predetermined failure threshold allowed an assessment of failure wind speeds.

For the first part of the project (estimating the pressure associated with a given wind speed), a simple load-response modelling framework was utilised. Given the inherent complexity of fluctuating wind loads on buildings, a simplified 'code-based' approach has been adopted for describing the load applied to the large door. This represented the wind load as a uniformly distributed normal load over an isolated screen. Section 3 details the wind load modelling.

Sections 4 to 6 then describe mechanical models that were developed to estimate the screen deformation and relative load distribution. These include a mass-spring model (Section 4), several continuum models (Section 5) and a model based on commercially available finite element packages (Section 6). While fundamental structural differences exist between these models and an integrated screen door system, they all conceptually model the deformation of a sheet under an applied pressure force. Each model has its own strengths and weaknesses, but all are considered a reasonable approximation of the real situation.

Section 8 brings together the results from the wind load models and the screen deformation models. The wind speeds associated with exceeding the failure threshold of the 'tabs' holding the screens in place (Section 2) are discussed, but further work is required to calibrate the underlying mechanical models and so it would not be prudent to take these results at face value and apply 
them at this stage.

The generalised load and response models presented here provide a baseline framework for generating a set of information for Centor to provide their clients with guidance on when screen doors should be stowed to minimise the chance of failure. A much more detailed description of building orientation, door placement and incident wind turbulence would be required to obtain detailed wind load information for any specific case. However, the analysis approach presented here does provide a generalised estimate of failure wind speed to be used by Centor to inform their clients.

\section{Background}

Centor screen doors are designed to unroll horizontally from a spool, and to be rolled up again when not in use. Figure 1 is a schematic showing two designs used for Centor screens. As depicted in the figure, a vertical spool is on the left hand end while the right hand end is held clamped. A system of springs connected to the right hand end of the screen maintains horizontal tension throughout the screen regardless of how far open or closed it is. In the original screen design (Figure 1 left), these horizontal restraints were tensioned to about $120 \mathrm{~N}$ and were the only means by which the screen was fixed; the upper and lower boundaries were free to move.

With time, Centor found that large screens with no restraint on the upper and lower boundaries were prone to large out-of-plane deformations, either through wind loading or the impact of people/animals colliding with the screen. These deformations were unsightly and in some cases meant the screen would be pulled out of its guide. Notably, if these actions caused the load transferred to the springs maintaining horizontal tension to exceed $500 \mathrm{~N}$, then the springs would fail. This would then require the costly replacement or repair of the door system by the owner.

As a result of these problems, Centor developed a new screen where the upper 
Figure 1: Schematic of the original (old) and current (new) screen designs. In the original screen design, the screen is kept in place purely through a tensioning system that applied $120 \mathrm{~N}$ of horizontal tension throughout the screen. In the 'new' screen design, there is less horizontal tension (only $60 \mathrm{~N}$ ) but tabs are introduced at the top and bottom of the screen to prevent the screen from bowing out too far. These tabs are designed so that they take no load when the the screen is unloaded (there is some give in the system), but they are then the weakest points when the screen is loaded excessively.

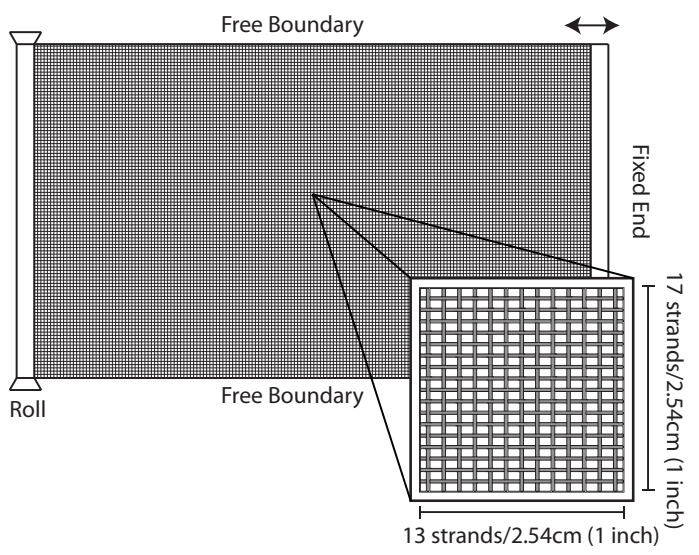

Original Design

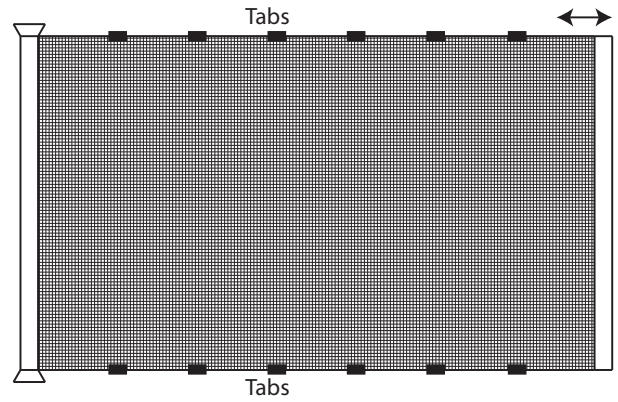

Current Design

and lower boundaries are constrained with intermittent tabs along the edges (Figure 2). In the design considered for this MISG project, upper and lower tabs are positioned directly above/below each other and are restrained in a guide rail system. In normal operation of the screen, tabs do not apply vertical tension to the screens as this would create unnecessary friction and impede the ability to slide the door smoothly. This 'frictionless' operation is achieved by manufacturing the screen with a vertical catenary so that gravity loading returns the screen to a perfect rectangular shape with the tabs (both upper and lower) resting loosely in the tracks. As such there is a small amount of 'give' in the screen before they are engaged. 
Figure 2: Example of a tab attached to the fabric of the screen. Failure occurs through the tab ripping apart from the main screen by pulling a few strands of fibre with them.

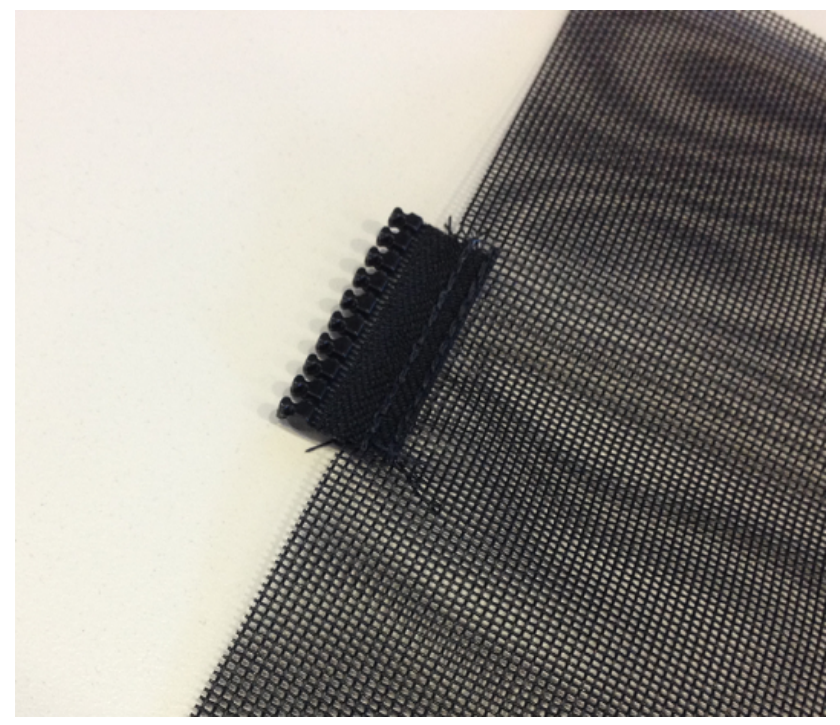

This new design has reduced the out-of-plane screen deformation and minimised the instances of horizontal tension spring failure by redistributing part of the applied load to the tabs. With this improvement though, a new type of failure has been observed; namely tearing of tabs from the screen itself. This is a much more desirable failure mode given that not all tabs are structurally necessary and a failed tab can easily be removed without the need to replace the screen completely.

Anecdotally, performance of the new screen design in response to wind loading is better than the performance of the old screen design. However, no information is available that would allow Centor to advise their customers about likely failure wind speeds for their doors. The Centor MISG team was tasked with estimating potential failure wind speeds for the screen door system so this information could be provided to Centor's customers. 
To facilitate this, prior to (and during) the MISG week, Centor measured important material properties that aided the mathematical modelling of their screens. Firstly, they found that the applied load required to cause an individual tab to fail was around $350 \mathrm{~N}$. The mode of tab failure was tearing of the plastic bond that holds individual strands of the screen mesh together. Secondly, Centor measured the load response of sections of screen material under tension (both under horizontal load and under vertical load). These results were quite noisy, but they led to the result that the horizontal and vertical strands are essentially identical, and the spring constant of a single strand is around $200 \mathrm{~N}$.

Lastly, Centor provided information about the macroscopic structure of their screens. The screen we model is in the form shown in Figure 1, with dimensions of $3 \mathrm{~m}$ high and $5 \mathrm{~m}$ wide. In the case where tabs are included in the model (as in Figure 1 right), there are six uniformly-spaced discrete tabs, each approximately $5 \mathrm{~cm}$ in length, placed along the top and bottom edges. Each screen is made up of strands with a diameter of $0.457 \mathrm{~mm}$. The strands are woven together so that there are 670 strands per metre in the horizontal direction (i.e., this is the density of the strands that run top to bottom) and 510 per metre in the vertical direction (i.e., this is the density of the strands that run left to right). This information forms the basis for the calculations of the deflection of the screens, and the approximated load on the tabs and springs.

\section{Determining the wind load on a screen door}

The distribution of wind loads on a building is a complex science. The magnitude (and even sign) of local pressures experienced by any given element, say a door, is dependent not only on the approach wind conditions (speed and turbulent structure), but also the building geometry and location of that element on the building. It is also variable with time. As such, much of the understanding of how wind loads buildings has come from wind tunnel 
experimentation and not direct numerical modelling [7]. Some research has been carried out in the computational domain [4], but the spectrum of turbulent scales requiring simulation still limits its application in practice.

The net load applied to any building face or element is a time varying combination of external and internal wind pressures applied to that element. External pressures are predominantly positive (pushing the face into the building) on the windward face of a building and negative on the side, back and roof faces. Internal pressures are either positive or negative depending on the location of opening/s on a building. In general the 'worst' loading combination for elements on a windward wall tend to occur when there is a large opening on the side or back walls, and conversely for the side, roof or back walls when there is a large opening on the windward wall.

The majority of existing wind tunnel studies focused on understanding the loading of buildings with impermeable surfaces. The exact case of loads on a large permeable door affixed to a predominantly impermeable building has not, as far as can be ascertained, been previously investigated in any great detail. However, research does exist that investigates the loads on simple porous fabric structures in isolation, such as wind breaks and signage [13, 9, 5]. These works show that the effect of porosity on the net wind load applied to these structures is an almost linear decrease in applied load as the structure's porosity (open area per unit total area) increases. Some dispute whether this is strictly a linear relationship, and whether the shape of porosity significantly changes the loss characteristics, but all researchers agree that as porosity increases the applied load decreases due to the greater ability of wind to flow through the structure.

In experiments that more closely resemble the current MISG problem, Robertson et al. [14] investigated the pressure distribution over permeable and impermeable greenhouses. Internal and external pressures were investigated for two different permeable fabrics and one impermeable membrane. Comparing results Robertson et al. [14] showed that external pressures on the windward face remained similar for all structures, but the suction pressures 
on the roof were significantly reduced when the cladding was permeable. Cheung and Melbourne [6] found similar results when investigating loads on a model building with impermeable walls but a porous roof. Robertson's experiments [14] went on to also show that internal pressures measured within the permeable greenhouse reduced when compared with the impermeable case.

Considering these wind tunnel studies it appears that changes in both external and internal pressure occur when buildings have permeable surfaces, or parts thereof, which significantly alter the net load on that surface. Unlike for the isolated wind breaks or signage, which showed a marked decrease in applied load as the porosity increased, limited, if any, reduction in load was observed for a porous windward face of a partially enclosed building. This is because the flux of air through the building face is considerably reduced when compared with the wind break or sign. In contrast, suction pressures on the roof appear to be reduced when that surface is porous [6]. A similar reduction in suction pressures would occur for a porous side wall given the similar nature of its loading profile. Further research is needed in this area, and approximations of how the porosity of the large door under investigation influences the load applied to it have had to be made.

Given time constraints of the MISG it was not possible to undertake a wind tunnel experiment or numerical simulation to quantify the loads on the large Centor door when located on a building. As such a simplified 'code-based' approach was adopted to determine input wind loads to be applied to the mathematical models. The Australian wind loading standard, AS/NZS1170.2 [1] was used and two example scenarios were defined for analysis. In the first scenario we assume that the Centor door is positioned on the windward face of a generic square building sited on flat ground, and several openings exist on the side and/or back walls of the building. This is notionally the worst-case geometric setup for loading of the door. In the second case the same building and door arrangement is considered, but the home is sited on top of a steep hill; this is a situation noted by Centor to have led to failures in the past. A schematic of the building geometry is shown in Figure 3. In accordance with AS/NZS1170.2 wind load is assumed to be uniformly and normally applied to 
Figure 3: Schematic diagram of assumed building, door and openings geometry.

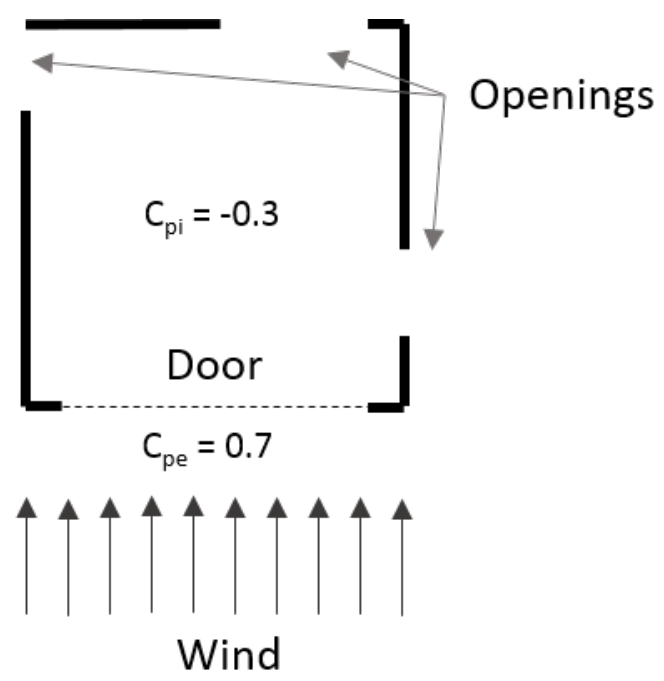

the door. In reality this is an oversimplification, but given the constraints of the project is considered reasonable.

Each of the mathematical models relate incident wind to the load applied to the door in a different way. Irrespective of the approach, AS/NZS1170.2 provides a method for calculating an equivalent oncoming mean wind speed, $V_{s}$, for each scenario. This method takes into account the geometric and topographic setup of the building and determines an equivalent wind speed considering the pressure used in Sections 4 to 6 to calculate the response of the screen to wind loading. It is possible to do this because no physical change to the structure of the loading profile (only the magnitude) is assumed in this approach.

Bernoulli's law is critical in analysis of the load associated with a given wind speed. This relates the pressure, $\mathrm{P}$, associated with a wind velocity, $\mathrm{V}$, by

$$
\mathrm{P}=\frac{\rho_{\text {air }} \mathrm{V}^{2}}{2},
$$


where $\rho_{\text {air }}$ is the density of air.

Making some simplifications to the equations provided in AS/NZS1170.2, the modelled gust wind speed, $\mathbf{V}_{\mathrm{m}}$, used in Sections 4 to 6 is converted to an equivalent scenario-relevant mean wind speed,

$$
V_{s}=\frac{1}{M_{t} G}\left[\frac{V_{m}^{2}}{\left(1-\beta_{s}\right)\left(C_{p e}-C_{p i}\right) K_{l}}\right]^{1 / 2}
$$

In this equation, $\mathrm{G}$ is a mean to peak wind speed adjustment (taken as 1.9), $\beta_{s}$ is the effective screen porosity assumed in the scenario, $M_{t}$ is a topographic hill shape multiplier, $K_{l}$ is a local pressure amplification factor, $C_{p e}$ is the external pressure coefficient and $C_{p i}$ is the internal pressure coefficient. As depicted in Figure 3 for the two scenarios considered $C_{p e}=0.7$ and $C_{p i}=$ -0.3 . For Scenario $1 M_{t}=1.0$, but for Scenario $2 M_{t}=1.5$, which corresponds with the building being situated on the top of a steep hill.

The mean to peak adustment, G, is required because AS/NZs1170.2 assumes a peak wind speed (averaging time of 0.2 seconds) and its resultant loading profile. Given this sort of information will seldom be available to Centor or their clients, an adjustment is introduced so a mean wind speed, similar to what is provided in a weather forecast, can be identified. The local pressure amplification factor $\mathrm{K}_{\mathrm{l}}$ is assumed to be 1.5 and represents the increase in localised wind pressure experienced when a restraint (the tab in this case) has a small tributary area. This increase in load arises because small scale turbulent eddies generate high localised pressures that influence only a portion of the door at any given point in time. In both scenarios $\beta_{s}$ is assumed to be 0 because the door is positioned on the windward face and Robertson et al. [14] showed little influence of porosity on the net load on this face. Unlike with isolated signage, the exact role that porosity plays in modifying surface pressures when the porous surface is positioned on a building is unclear.

In short, we find that it is useful to consult codes such as AS/NZS1170.2 in order to determine the 'equivalent wind speed' associated with a given house geometry and wind loading conditions. Equation (2) shows how the gust wind 
speed is used to estimate the effective wind speed if parameters associated with geography and pressure amplification can be determined. In many cases, these parameters will be difficult to estimate: more work, in particular, is needed to determine the role of porosity, since most of the wind codes were developed to determine the load on a solid surface rather than on a porous screen. In much of the work that follows, Equation (1) is used to determine wind load. However, in Section 8, Equation (2) is applied to select mechanical model output to exemplify its use through Scenarios 1 and 2.

\section{Mass-spring models for the deformation of a screen}

\subsection{Developing a mass-spring model for the deformation of a door}

Within this section, we present a simple elastic model of the screen structure, based on the work of Provot [12]. Rather than treating the structure as a continuous sheet, we approximate it as a series of masses connected by springs, and model the force interactions between each mass. This has a number of strengths and weaknesses. Some strengths of this approach include that it is not necessary to translate the wind speed into a pressure (it is simply applied as a drag on each mass), that different geometries (the untabbed and tabbed screens in Figure 1) are easy to deal with, and that it is easy to interpret the results in terms of both deflection and the load on the tabs. At the same time, the mass-spring approach suffers from high computational cost (although not prohibitive) and the necessity to introduce additional 'fictional' springs to account for the manner in which strands will resist sharp angles at junctions.

Mathematically, we discretise the screen structure into a mesh of $m \times n$ virtual masses, placed at each junction of two fibers. Each mass is denoted by the subscript $[i, j]$, with $i=1, \ldots, \mathfrak{m}, \mathfrak{j}=1, \ldots, \mathfrak{n}$. The masses are then 
linked together by massless springs of a natural, non-zero length. Three types of spring linkages are applied (Figure 4).

- The mass $[i, j]$ is connected to its horizontal and vertical neighbours $[i+$ $1, j]$ and $[i, j+1]$ by structural springs.

- The mass $[i, j]$ is connected to its diagonal neighbours $[i+1, j+1]$ and $[i+1, j-1]$ by shear springs.

- The mass $[i, j]$ is connected to its secondary horizontal and vertical neighbours $[i+2, j]$ and $[i, j+2]$ by flexion springs.

The shear and flexion springs are not physically present in the screen structure. However, we include these artificial linkages to account for the shear and flexion forces that the structure undergoes during deformation.

\subsection{Force balance equations for the mass-spring model}

To model the deformation of the structure over time, we consider the forces that are being applied to it. We first define $\mathbf{P}_{i, j}(t)$ to be the position vector of mass $[i, j]$ at time $t$, with coordinates $\left(x_{i, j}(t), y_{i, j}(t), z_{i, j}(t)\right)$. Using Newton's Second Law of Motion,

$$
F_{i, j}=\mu a_{i, j},
$$

where $\mu$ is the mass of each junction (considered equal), and $\boldsymbol{a}_{i, j}$ is the acceleration of the mass $[i, j]$ caused by the force $\mathbf{F}_{i, j}$.

The force $\boldsymbol{F}_{i, j}$ is comprised of four major forces [12]. The first is the internal force $F_{i n t}$, which results from the tension of the springs attached to mass $[i, j]$. Considering Hooke's Law,

$$
\mathrm{F}_{\text {int }}\left(\mathrm{P}_{i, j}\right)=-\sum_{\text {linkages }} \mathrm{K}_{i, j, k, l}\left[\mathbf{l}_{i, j, k, l}-\mathrm{l}_{i, j, k, l}^{0} \frac{\mathbf{l}_{i, j, k, l}}{\left\|\mathbf{l}_{i, j, k, l}\right\|}\right],
$$

where 
Figure 4: An example of the artificial meshing structure, showing structural, shear, and flexion springs, based on the approach used by Provot [12]. Masses (shown as circles) are connected by springs if there is a line that begins at one mass and terminates at the other.

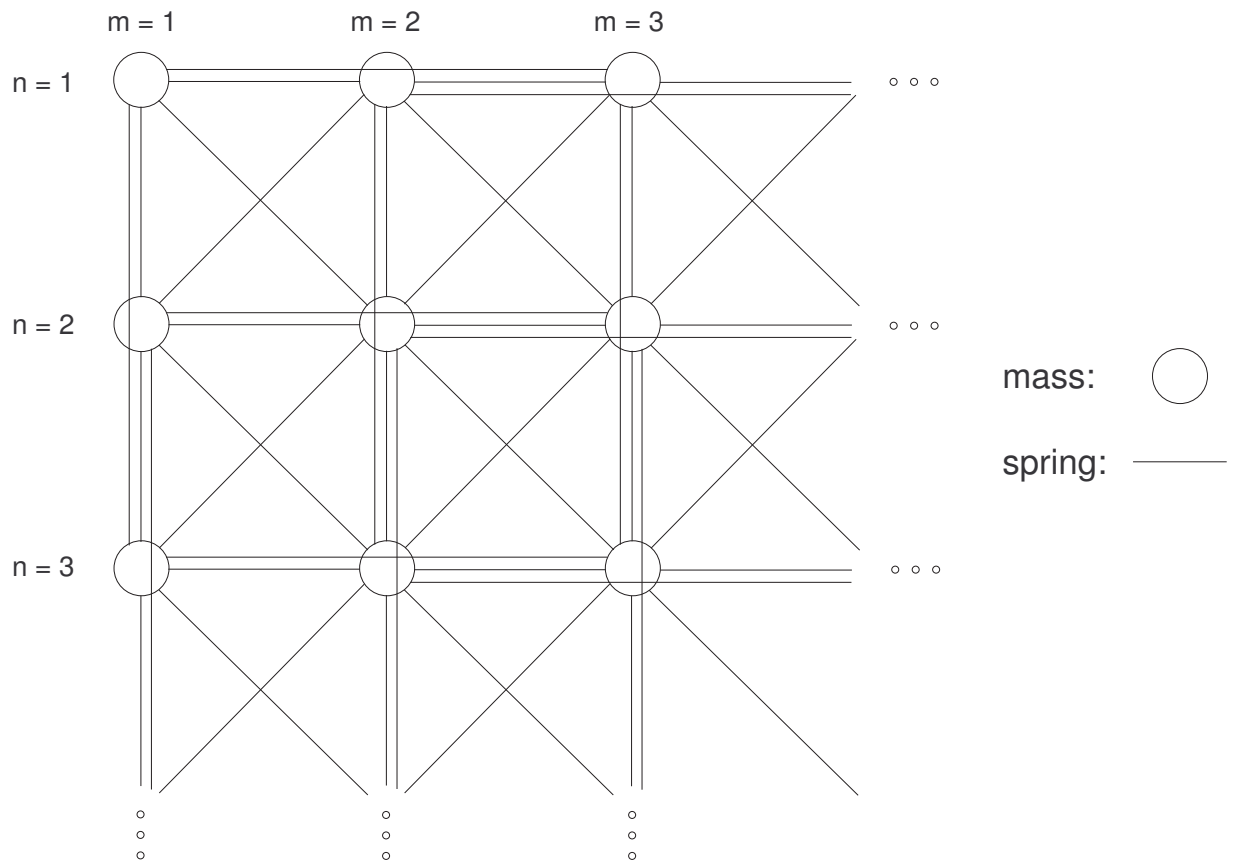

- each $(k, l)$ pair is taken from the set of masses linked to mass $[i, j]$,

- $\mathbf{l}_{\mathrm{i}, \mathrm{j}, \mathrm{k}, \mathrm{l}}=\mathbf{P}_{\mathrm{k}, \mathrm{l}}-\mathbf{P}_{\mathrm{i}, \mathrm{j}}$,

- $l_{i, j, k, l}^{0}$ is the natural length of the spring linking $\mathbf{P}_{i, j}$ and $\mathbf{P}_{k, l}$, and

- $\mathrm{K}_{i, j, k, \mathrm{l}}$ is the stiffness coefficient of the spring linking $\mathbf{P}_{i, j}$ and $\mathbf{P}_{k, l}$.

Alongside this force, there are three major external forces: a gravity load, viscous damping, and an interaction with an air stream. Taking the acceleration 
due to gravity as $\mathbf{g}$, the gravity force is

$$
\mathrm{F}_{\mathrm{gr}}\left(\mathbf{P}_{\mathrm{i}, \mathrm{j}}\right)=\mu \mathrm{g} .
$$

The viscous damping is

$$
F_{\text {damp }}\left(\mathbf{P}_{i, j}\right)=-C_{\text {damp }} \boldsymbol{v}_{i, j},
$$

where $C_{\text {damp }}$ is a damping coefficient, and $\boldsymbol{v}_{i, j}$ is the velocity of the mass $[\boldsymbol{i}, \boldsymbol{j}]$. While not necessarily an external force, this force is included to reconcile the dissipation of mechanical energy in the system. Without damping, the screen would continue to oscillate forever in response to a change of load.

Finally, the force applied to the structure by a wind moving at a constant velocity, $\mathbf{u}$, follows from Equation (1),

$$
\mathbf{F}_{\text {air }}\left(\mathbf{P}_{i, j}\right)=\frac{\rho_{\text {air }} A_{i, j}}{2}\left[\mathbf{n}_{i, j} \cdot\left(\mathbf{u}-\boldsymbol{v}_{i, j}\right)^{2}\right] \mathbf{n}_{i, j},
$$

where $\mathbf{n}_{i, j}$ is the unit normal of the surface at $\mathbf{P}_{i, j}$, the superscript represents that each component of the vector is squared, and $A_{i, j}$ is the surface area associated with mass $[i, j]$ (given by the total door area divided by the number of masses).

Considering the total resultant force $\mathbf{F}_{i, j}$, we explicitly integrate Newton's Second Law using a Forward Euler Method, to give

$$
\begin{aligned}
& a_{i, j}(t+\delta t)=\frac{1}{\mu} F_{i, j}(t), \\
& v_{i, j}(t+\delta t)=v_{i, j}(t)+\delta t a_{i, j}(t+\delta t), \\
& P_{i, j}(t+\delta t)=P_{i, j}(t)+\delta t v_{i, j}(t+\delta t),
\end{aligned}
$$

where $\delta \mathrm{t}$ is the time-step size. 
Figure 5: Representation of the screen door mesh. The screen is fixed on the left and right boundaries, as well as at specified tab points on the top and bottom boundaries.

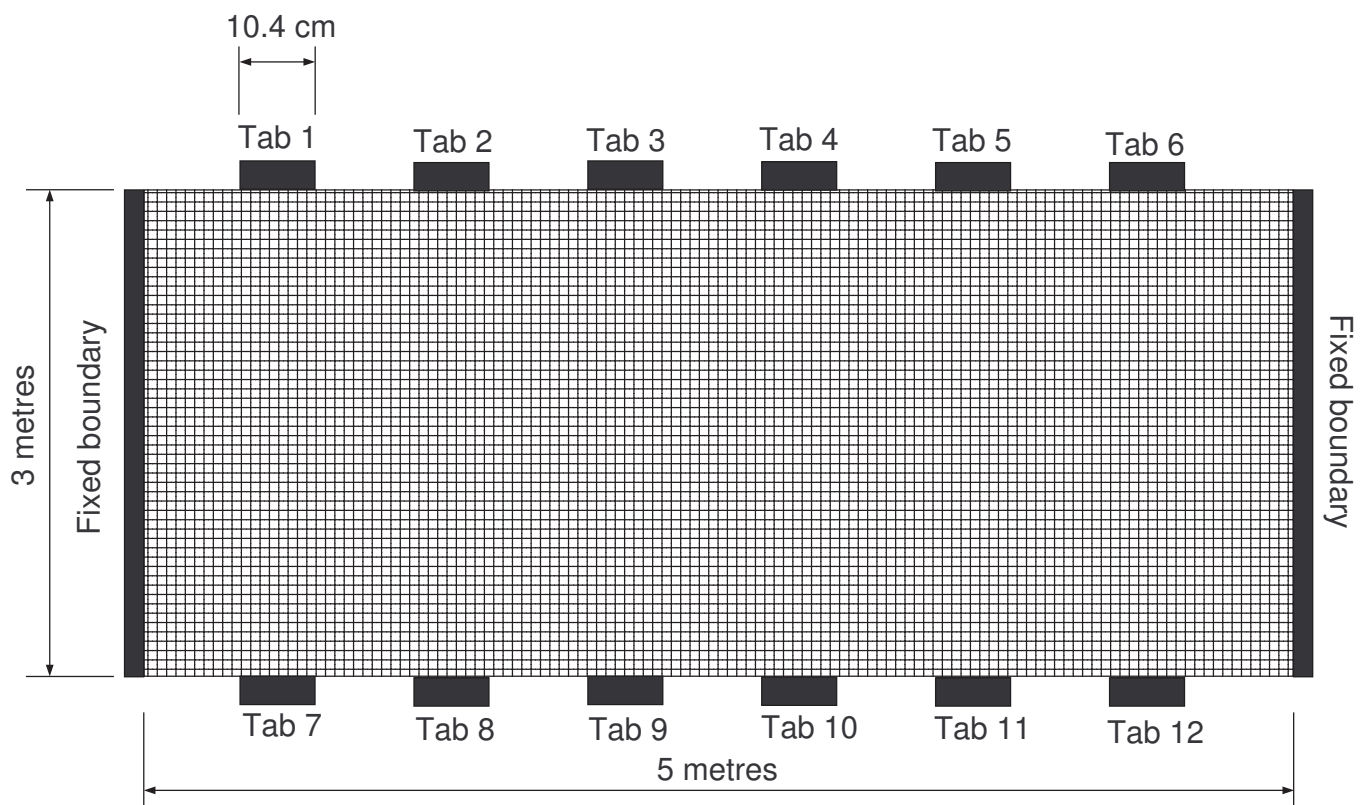

\subsection{Results from the mass-spring model}

In this section we consider the results from the mass-spring model. The focus is on investigating the forces distributed across each of the tabs (as shown in Figure 5) and the left and right hand sides of the screen. We assume that the screen is fixed on the left and right boundaries and at the tabs. The tabs are evenly distributed with a length of $10.4 \mathrm{~cm}$ and are aligned on the top and bottom boundaries.

We consider the loads as caused by seven wind speeds: 20, 40, 60, 80, 100, 120 and $140 \mathrm{~km} / \mathrm{h}$. The wind is oriented so as to be travelling perpendicular to the surface of the screen. 
Figure 6: Total force $(\mathrm{N})$ applied to each of the tabs on the screen door and the side boundaries for wind speeds of $20 \mathrm{~km} / \mathrm{h}$. The numbering of the tabs is as presented in Figure 5.
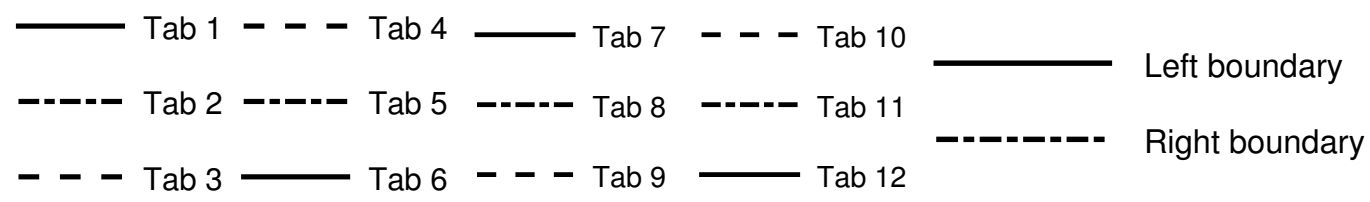

(a) Tabs 1-6.

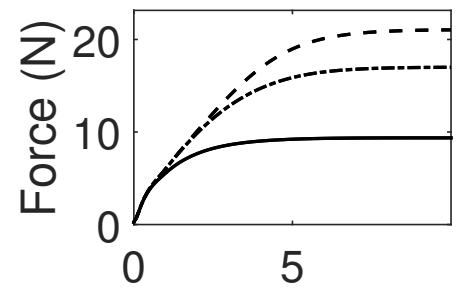

Time (s) (b) Tabs $7-12$.

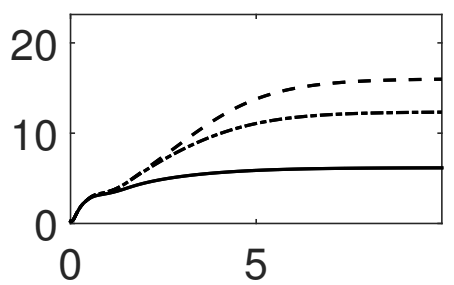

Time (s) (c) Side boundaries.

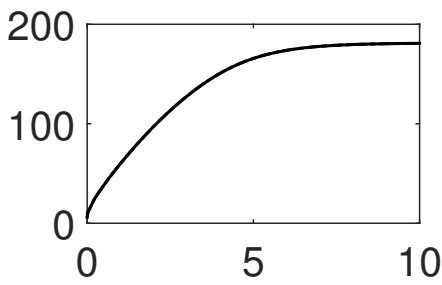

Time (s)

Figure 7: Total force $(\mathrm{N})$ applied to each of the tabs on the screen door and the side boundaries for wind speeds of $40 \mathrm{~km} / \mathrm{h}$. The numbering of the tabs is as presented in Figure 5.

(a) Tabs 1-6.

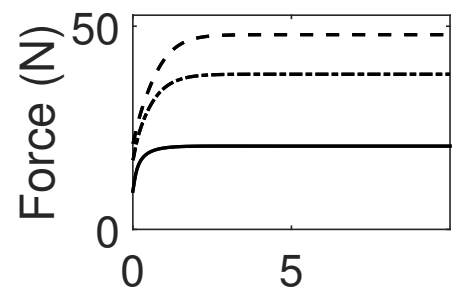

Time (s) (b) Tabs 7-12.

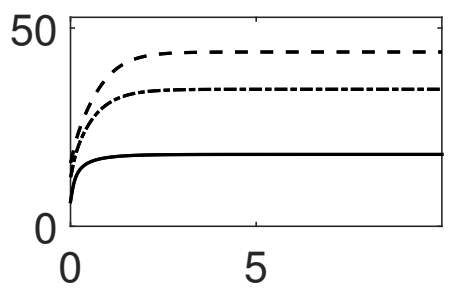

Time (s) (c) Side boundaries.

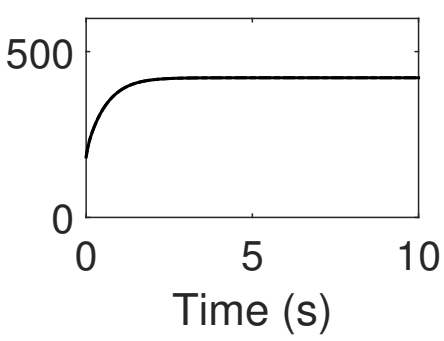


Figure 8: Total force $(\mathrm{N})$ applied to each of the tabs on the screen door and the side boundaries for wind speeds of $60 \mathrm{~km} / \mathrm{h}$. The numbering of the tabs is as presented in Figure 5.

(a) Tabs 1-6.

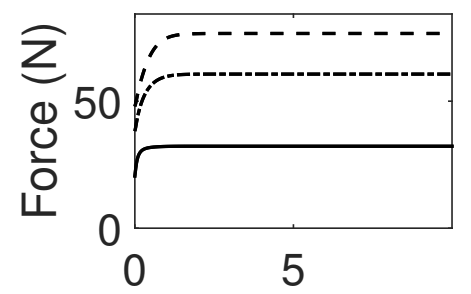

Time (s) (b) Tabs 7-12.

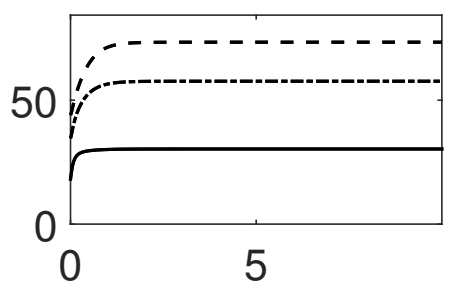

Time (s) (c) Side boundaries.

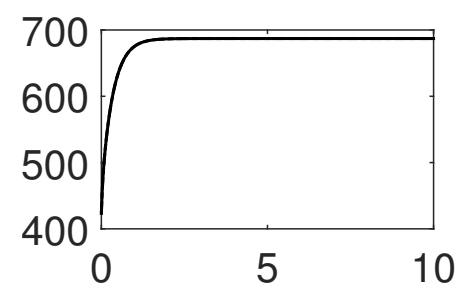

Time (s)

Figure 9: Total force $(\mathrm{N})$ applied to each of the tabs on the screen door and the side boundaries for wind speeds of $80 \mathrm{~km} / \mathrm{h}$. The numbering of the tabs is as presented in Figure 5.

(a) Tabs 1-6.

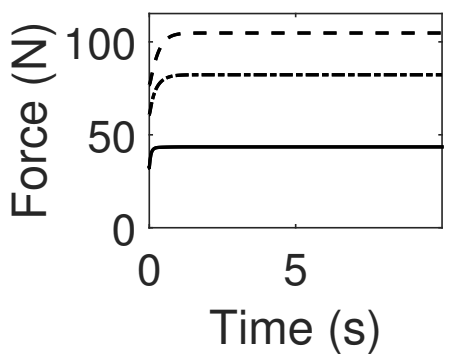

(b) Tabs 7-12.

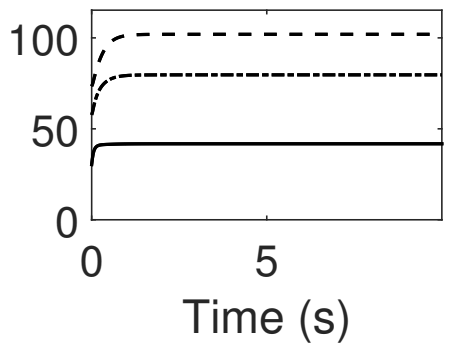

(c) Side boundaries.

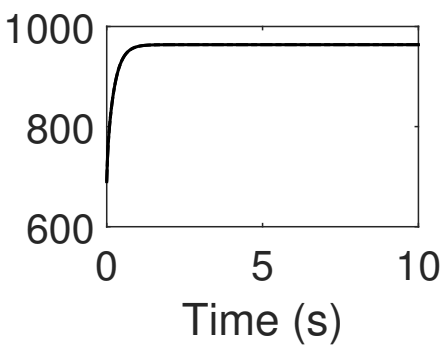


Figure 10: Total force $(\mathrm{N})$ applied to each of the tabs on the screen door and the side boundaries for wind speeds of $100 \mathrm{~km} / \mathrm{h}$. The numbering of the tabs is as presented in Figure 5.

(a) Tabs 1-6.

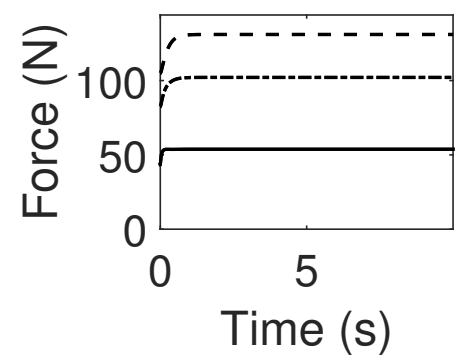

(b) Tabs 7-12.

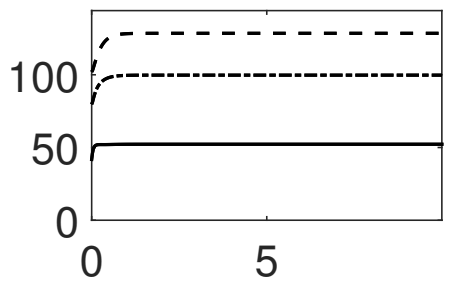

Time (s) (c) Side boundaries.

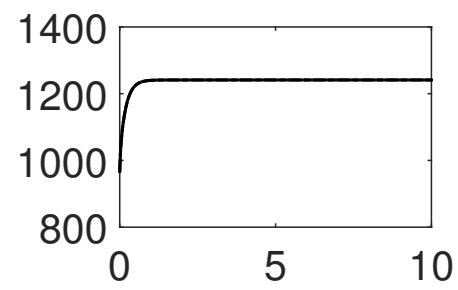

Time (s)

Figure 11: Total force $(\mathrm{N})$ applied to each of the tabs on the screen door and the side boundaries for wind speeds of $120 \mathrm{~km} / \mathrm{h}$. The numbering of the tabs is as presented in Figure 5.

(a) Tabs 1-6.

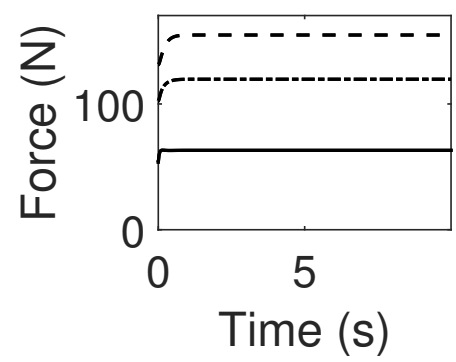

(b) Tabs 7-12.

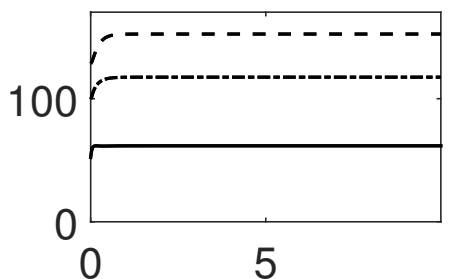

Time (s) (c) Side boundaries.

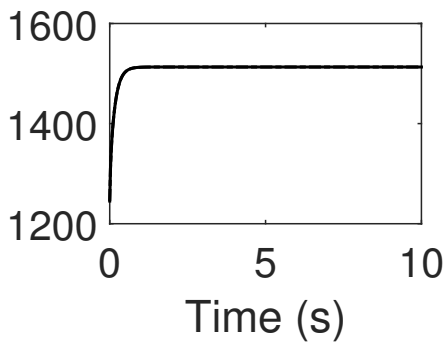


Figure 12: Total force $(\mathrm{N})$ applied to each of the tabs on the screen door and the side boundaries for wind speeds of $140 \mathrm{~km} / \mathrm{h}$. The numbering of the tabs is as presented in Figure 5.

(a) Tabs 1-6.

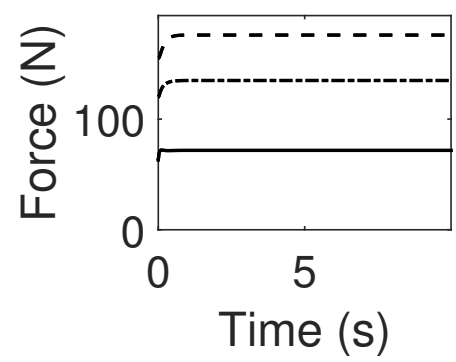

(b) Tabs 7-12.

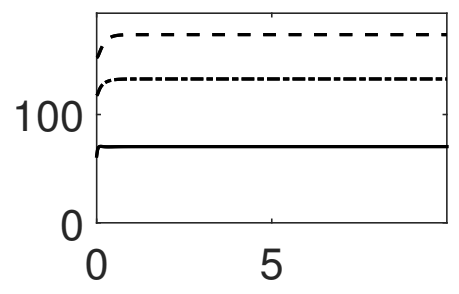

Time (s) (c) Side boundaries.

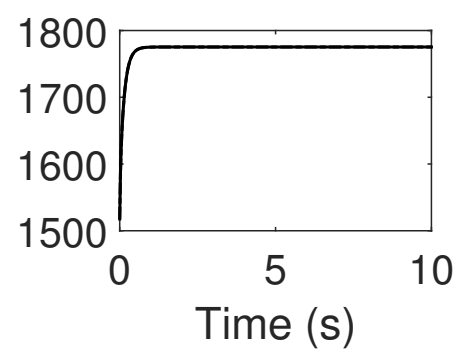

Figures 6 to 12 show the total force that is applied to each of the tabs attached to the top and bottom boundaries of the screen door, as well as the left and right boundaries, when subjected to a constant wind speed. The force exerted on the top tabs is consistently larger than that on the bottom tabs. This is due to the effect of gravity that acts in tandem with the spring forces on the top tabs as the screen door is stretched. On the other hand, gravity would counteract the force exerted by the springs on the bottom tabs.

These results indicate that the greatest force is exerted on the tabs located near the centre of the screen (Tabs 3 and 4 at the top, and Tabs 9 and 10 on the bottom). Due to symmetry of the screen used in this model, the forces applied to the pairs of tabs from the centre of the screen is identical (including the left and right boundaries).

In addition to tab distributed forces, Table 2 and Figure 13 show the simulated maximum deflections, together with a fitted function for a range of wind speeds. The relationship between wind speed and maximum deflection is well characterised by a power law of the form $d_{\max }=a w^{b}$, where $d_{\max }$ is the maximum deflection, $w$ is the wind speed, and $a=0.066$ and $b=0.68$ are curve parameters obtained by fitting to the simulated deflections. Deformations are larger than one may expect, suggesting some level of calibration of 
Table 1: Maximum total force exerted on tabs and boundaries for a range of wind speeds.

\begin{tabular}{c|rrrrrrr}
\hline & \multicolumn{7}{|c}{ Wind speed $(\mathrm{km} / \mathrm{h})$} \\
Tabs & 20 & 40 & 60 & 80 & 100 & 120 & 140 \\
\hline 1 and 6 & 9 & 21 & 32 & 43 & 54 & 63 & 72 \\
2 and 5 & 17 & 38 & 61 & 82 & 102 & 120 & 135 \\
3 and 4 & 21 & 48 & 77 & 105 & 131 & 152 & 176 \\
7 and 12 & 6 & 18 & 30 & 42 & 52 & 62 & 71 \\
8 and 11 & 12 & 35 & 58 & 80 & 100 & 117 & 133 \\
9 and 10 & 16 & 44 & 73 & 102 & 129 & 153 & 174 \\
$\quad$ Sides & 181 & 421 & 687 & 964 & 1241 & 1513 & 1775 \\
\hline
\end{tabular}

Table 2: Deflection of the screen for a range of wind speeds.

\begin{tabular}{cc}
\hline Wind speed $(\mathrm{km} / \mathrm{h})$ & Maximum Deflection $(\mathrm{m})$ \\
\hline 20 & 0.50 \\
40 & 0.80 \\
60 & 1.05 \\
80 & 1.28 \\
100 & 1.49 \\
120 & 1.69 \\
140 & 1.88 \\
\hline
\end{tabular}


Figure 13: Simulated and fitted maximum deflections of the screen for a range of wind speeds.

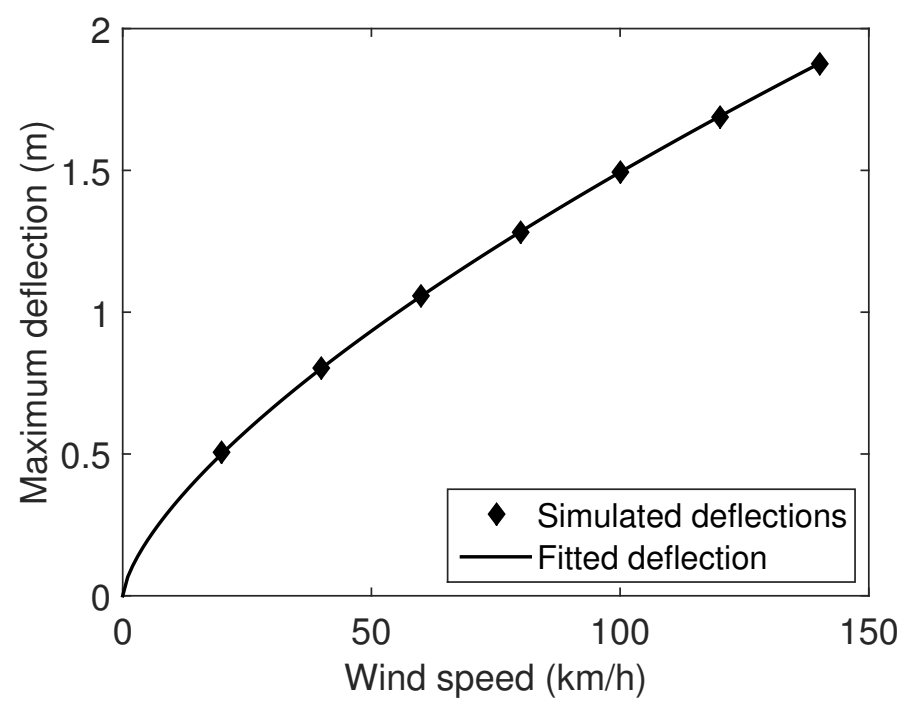

spring constants is required. Results discussed in Section 5.4 confirm this to be the case.

\section{Continuum models for the deformation of a screen}

\subsection{General background to membrane models}

Throughout this section, we treat the screens developed by Centor as continua. That is, we do not attempt to find the deformations of the individual strands that make up the screens, but instead consider the averaged mechanical properties on a macroscopic scale. This leads to partial differential equations for the perpendicular displacements of the screen as a function of position in 
the unloaded state.

While we do not solve for the deformations of individual strands, the fibrous nature of the screen is still important in determining the form of the model and the associated model parameters. Since the screens are composed of strands running in the vertical and horizontal directions (with different strand densities in the two different directions), any continuum model of a screen should be orthotropic, with the principal axes of the mechanical properties aligned with the warp and weft directions of the woven screen. Moreover, the continuum coefficients of elasticity in these directions are obtained from the strand density and the elastic moduli of the individual strands, as discussed in Section 2.

Since the strands that make up the screen have relatively low resistance to bending, but there is a significant horizontal tension in the screen, it is more appropriate to use a membrane model than a plate model [8]. Physically, this corresponds to the fact that a screen will behave more like a drumskin held under tension than a sheet of metal - the main resistance to deformation is due to the tension in the screen rather than any bending stiffness.

The classical membrane equation is stated conveniently in variational form. Given a membrane with horizontal tension per unit length $\mathcal{T}_{x}\left(\mathrm{Nm}^{-1}\right)$ and vertical tension per unit length $\mathcal{T}_{y}\left(\mathrm{Nm}^{-1}\right)$, both of which are constant but not necessarily equal, finding the perpendicular deflection of a membrane $w(x, y)$ due to a spatially-varying load $f(x, y)$ is equivalent to minimising the functional

$$
\iint_{R} \frac{\mathcal{T}_{x}}{2}\left(\frac{\partial w}{\partial x}\right)^{2}+\frac{\mathcal{T}_{y}}{2}\left(\frac{\partial w}{\partial y}\right)^{2}-f(x, y) w(x, y) d A
$$

subject to appropriate boundary conditions on $w(x, y)$, where $R=[0, W] \times$ $[0, \mathrm{H}]$ is the region occupied by the screen (assumed to be of width $W$ and height $\mathrm{H})$.

In the case of the 'original' screens (without tabs holding them in place), the 
appropriate boundary conditions on $w(x, y)$ are

$$
w(0, y)=w(W, y)=0, \quad w_{y}(x, 0)=w_{y}(x, H)=0 .
$$

This corresponds to the physical situation where there is no perpendicular displacement of the screen on the left or right hand sides, but where the top and bottom boundaries are free to move.

In the case where tabs are present, the latter boundary condition (associated with the top and bottom boundaries) needs to be adapted. The simplest way to achieve this is to assume that $w(x, 0)=w(x, H)=0$ wherever a tab is present while $w_{y}(x, 0)=w_{y}(x, H)=0$ in the regions without tabs. This corresponds to a situation where the tabbed regions are fixed (just like the left and right boundaries), while the untabbed regions are free to move as before.

Unfortunately, this is not strictly accurate. The Centor screens are designed so that there is some give in them before the tabs take any load. That is, it is possible for there to be some displacement in the tabbed regions before the effect of the tabs is felt. As discussed in Section 5.2, this leads to the possibility of using alternative boundary conditions that incorporate the 'give' in the tabs.

An additional issue with the functional (11) arises from there being be no vertical tension in the system initially. However, as the screen deforms under load, the associated stretch in the vertical strands leads to the emergence of vertical tension that resists further deformation. A further, and related, issue is that the classical membrane equation assumes that the horizontal tension across the screen is constant, regardless of the deformation: as a result, it is never possible to use the classical membrane equation to describe a failure of the screen where the horizontal tension under wind load exceeds the critical stress of the springs keeping the sheet under tension. In order to deal with both of these issues, the tension throughout the screen is made a function of the deformation.

This modification of the classical equations of elastic strings and membranes is 
called 'tension modulation', and models of screen deformation that incorporate tension modulation are described in Section 5.3. The problem of tension modulation is often considered in acoustics, since tension modulation can have a significant effect on the timbre and pitch associated with the vibrations of a string or membrane. There is a significant literature on how to incorporate tension modulation into models of vibrating strings $[3,11,15$, e.g.] and vibrating membranes [2, e.g.]. By extension, the same models can be used to determine the deflection of a loaded membrane when tension modulation is important.

A further issue that is ignored in (11) is that the perpendicular deflections of the screens under load have the potential to be significant. The membrane equation (11) is derived by assuming that all displacements are small; however, Centor observe that their screens can bow significantly (with displacement of up to $30 \mathrm{~cm}$ ) before any damage occurs to the springs or tabs. Assuming that the deformations are large but that the strains are small, it is possible to derive geometrically-nonlinear expressions for the deformation of a membrane or plate. The classic example of a geometrically-nonlinear plate is a diving board: while someone bouncing on the end of a diving board leads to significant displacements of the end of the board, the curvature of the board at any particular point remains fairly small. As a result, it is not necessary to consider nonlinearity in the definitions of elastic energy, but it is necessary to consider the role of geometric nonlinearities in calculating strain.

One area in which geometric nonlinearity in the deflection of a membrane is important is in the calculation of the shape of the cornea in response to the load due to intraocular pressure. While apparently very different from the deflection of a screen door in response to wind loading, both situations involve the deflection of a membrane due to a load from applied pressure, and so geometrically nonlinear membrane models originally developed to describe the shape of the cornea [10, e.g.] can be applied directly to modelling the deflection of screen doors in the wind. This leads to the results given in Section 5.4. 


\subsection{Membrane models without tension modulation}

In the complete absence of vertical tension (and without the tension modulation discussed in Section 5.3), the energy of a membrane degenerates to take the form of the energy of an elastic string:

$$
E[w(x, y)]=\iint_{R} \frac{T_{x}}{2 H}\left(\frac{\partial w}{\partial x}\right)^{2}-f(x, y) w(x, y) d A
$$

There is no energy penalty associated with large vertical gradients in $y$, and so this expression is unlikely to yield realistic solutions. In the case where we assume that $w$ is a function of $x$ only (as we do in Section 5.3.2), where $f(x, y)$ is constant, and where fixed boundary conditions are applied at $x=0$ and $x=W$, we minimise (13) to obtain the simple solution

$$
w(x, y)=\frac{f H}{2 T_{x}} x(W-x) .
$$

If we introduce a small amount of vertical tension to eliminate the degeneracy, then the membrane equation can be solved using Green's function methods or other classical solution techniques. For example, in the case where there is a small tension per unit length $\mathcal{T}_{\mathfrak{y}}=\epsilon \ll 1$ and $\mathrm{f}$ is constant, we find that writing

$$
w(x, y)=\frac{f H}{2 T_{x}} x(W-x)+\tilde{w}(x, y)
$$

leads to a situation where $\tilde{w}$ satisfies a rescaled form of Laplace's equation,

$$
-\frac{\mathrm{T}_{\chi}}{\mathrm{H}} \frac{\partial^{2} \tilde{w}}{\partial x^{2}}-\epsilon \frac{\partial^{2} \tilde{w}}{\partial y^{2}}=0,
$$

where the boundary conditions on $\tilde{w}$ are obtained from the boundary conditions on $w$.

In the simple (but unrealistic) case where we apply zero displacement boundary conditions on the top and bottom edges, this ultimately leads to an explicit 
solution for $\tilde{w}$ as a Fourier series expansion, yielding

$$
\begin{aligned}
& w(x, y)=\frac{f H}{2 T_{x}} x(W-x) \\
& +\sum_{n=1}^{\infty} \frac{4 W^{2}(-1)^{n} f T_{x} \cosh \left[\left(n-\frac{1}{2}\right) T_{x}^{\frac{1}{2}} \epsilon^{-\frac{1}{2}} H^{-\frac{1}{2}} \pi(2 y-H) / W\right] \sin [n \pi x / W]}{(2 n-1)^{3} \pi^{3} H \cosh \left[\left(n-\frac{1}{2}\right) T_{x}^{\frac{1}{2}} \epsilon^{-\frac{1}{2}} \pi H^{\frac{1}{2}} / W\right]}
\end{aligned}
$$

A solution based on three terms of this series with $\mathrm{W}=4 \mathrm{~m}, \mathrm{H}=2.7 \mathrm{~m}$, $\mathrm{T}_{\mathrm{x}}=60 \mathrm{~N}, \epsilon=0.25 \mathrm{~N} \mathrm{~m}^{-1}, \mathrm{f}=3 \mathrm{~Pa}$ (corresponding to a wind speed of around $8 \mathrm{~km} \mathrm{~h}^{-1}$ or $2.2 \mathrm{~m} \mathrm{~s}^{-1}$ by Bernoulli's law) is shown in Figure 14. The screen bows out significantly (up to $40 \mathrm{~cm}$ ) even with the very small applied load and the zero-displacement boundary conditions. With only small vertical tension, there is very little to prevent the screen from deflecting just as much as it would do in the absence of any vertical tension.

The large deflections observed with small vertical tension motivate us to improve the model. One possible approach to take is to introduce tension modulation, as discussed in Section 5.3; this would reduce the deflection because there would be an increase in tension associated with any increase in deformation. An alternative is to think more carefully about the restraint imposed by the presence of tabs. One idea would be to impose the tethering effects of the tabs as an inextensibility constraint in the vertical direction.

This approach has certain problems (particularly in that we are treating the strands that connect the vertical tabs as being inextensible while all other strands are treated as being elastic), but it has the advantage over other methods in that it leads to a very tractable mathematical problem (indeed, a problem with a closed form solution), and that it is very easy to obtain the force applied to the tabs from this solution.

Thus, consider the case where there is a small amount of give in the system, but where the strands connecting the tabs are close to being inextensible. Hence, if the tabs are taking load (which we assume), then the length of the 
Figure 14: Deflection of a screen governed by the membrane equation with fixed boundaries on all sides and minimal tension in the vertical direction, based on the Fourier series solution (17).

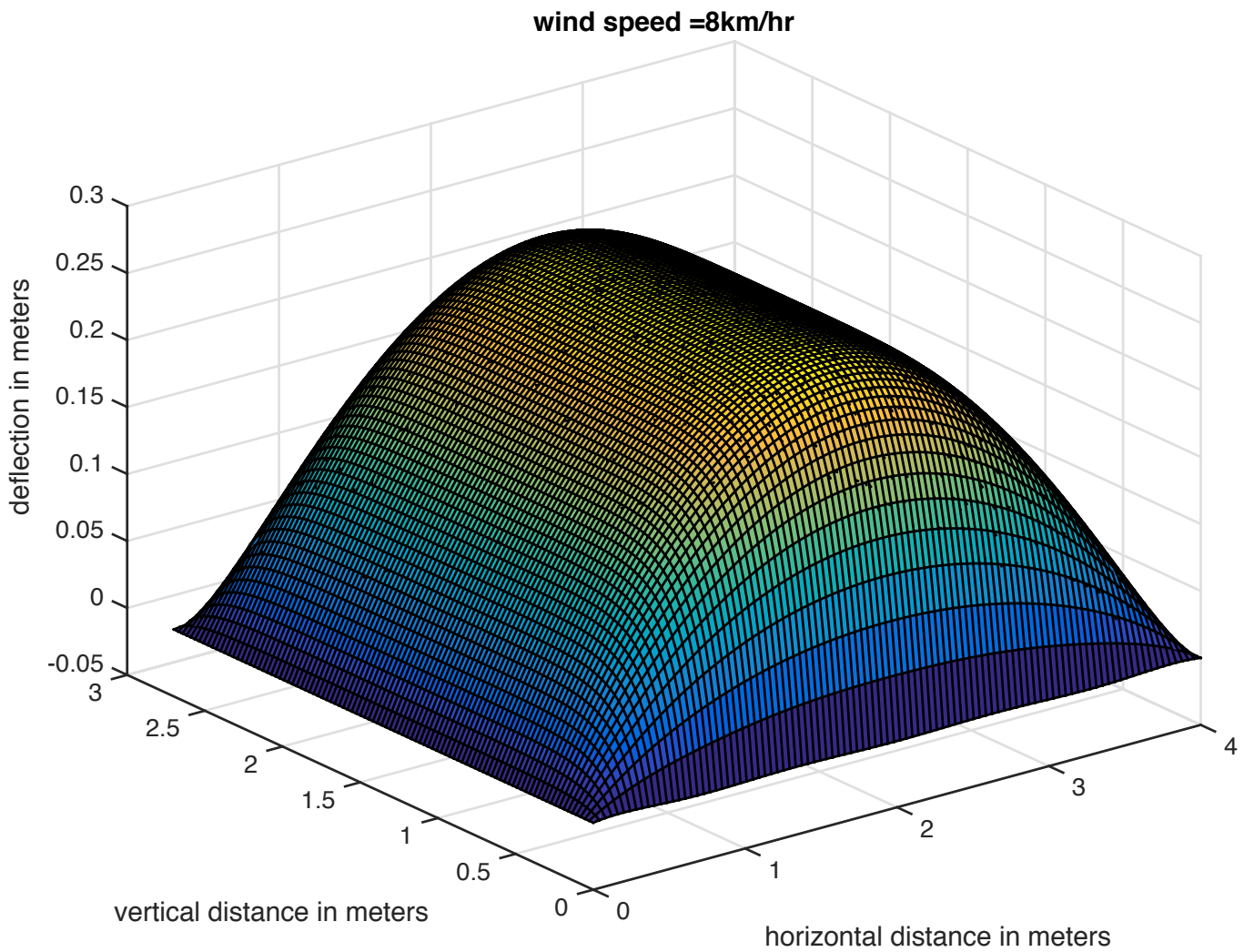


arc from one tab to its opposite neighbour is specified. This motivates us to construct a new energy functional that includes a Lagrange multiplier to impose the arclength constraint between tabs. Assuming no vertical tension for simplicity, this takes the form

$$
\begin{aligned}
E[w(x, y), \lambda]=\iint_{R} \frac{T_{x}}{2 H}\left[\frac{\partial w(x, y)}{\partial x}\right]^{2}-f(x, y) w(x, y) d A \\
+\sum_{i=1}^{n} \lambda_{i}\left\{\int_{0}^{H} \frac{1}{2}\left[\frac{\partial w\left(x_{i}, y\right)}{\partial y}\right]^{2} d y-\kappa\right\},
\end{aligned}
$$

where $x_{i}(m)$ represents the locations of the tabs; $\lambda_{i}(\mathrm{~N})$ are Lagrange multipliers representing the force on the tabs; and $\mathrm{\kappa}(\mathrm{m})$ represents the length of the 'give' in the system, so that the arc between $\left(x_{i}, 0\right)$ and $\left(x_{i}, H\right)$ has length $\mathrm{H}+\mathrm{K}$. The energy in (18) must be minimised subject to the boundary conditions

$$
w(0, y)=w(W, y)=0, \quad w\left(x_{i}, 0\right)=w\left(x_{i}, H\right)=0 \quad \text { for all } i
$$

The length constraints at $x=x_{i}$ are a little unusual, and a naïve attempt to minimise (18) by constructing the Euler-Lagrange equation fails because the system of equations associated with (18) has no strong solutions. To see this, we take a functional derivative of $E$ with respect to $w$ in the direction of $\eta(x, y)$, where $\eta$ obeys the boundary conditions in (19), to obtain

$$
\begin{aligned}
&\left.\frac{\partial E}{\partial w}\right|_{\eta}=\lim _{\epsilon \rightarrow 0} \frac{E[w+\epsilon \eta, \lambda]-E[w, \lambda]}{\epsilon} \\
&=\iint_{R} \frac{T_{x}}{H} \frac{\partial w(x, y)}{\partial x} \frac{\partial \eta(x, y)}{\partial x}-f(x, y) \eta(x, y) d A \\
& \quad+\sum_{i=1}^{n} \lambda_{i} \int_{0}^{H} \frac{\partial w\left(x_{i}, y\right)}{\partial y} \frac{\partial \eta\left(x_{i}, y\right)}{\partial y} d y .
\end{aligned}
$$


Integrating by parts and applying the boundary conditions on $\eta$, we therefore find that

$$
\begin{aligned}
&\left.\frac{\partial E}{\partial w}\right|_{\eta}=\iint_{R}\left[-\frac{T_{x}}{H} \frac{\partial^{2} w(x, y)}{\partial x^{2}}-f(x, y)\right] \eta(x, y) d A \\
&-\sum_{i=1}^{n} \lambda_{i} \int_{0}^{H} \frac{\partial^{2} w\left(x_{i}, y\right)}{\partial y^{2}} \eta\left(x_{i}, y\right) d y .
\end{aligned}
$$

Since this must be zero for all $\eta(x, y)$ at a minimiser of $E$, including choices of $\eta(x, y)$ where $\eta=0$ whenever $x=x_{i}$, we therefore find that the EulerLagrange equation is (as before)

$$
-\frac{\partial^{2} w(x, y)}{\partial x^{2}}-f(x, y)=0
$$

subject to boundary conditions $w(0, y)=w(W, y)=0$. However, the solutions obtained to this will not obey the length constraints (which could be obtained from (18) by differentiating $E$ with respect to the various components $\boldsymbol{\lambda}$ ), and hence we apparently reach a contradiction.

The reason for this apparent contradiction is that we made a mistake by assuming that $w_{x}(x, y)$ is continuous throughout the entire domain in order to obtain the Euler-Lagrange differential equation by integration by parts. Instead, we find that the minimisers of (18) must necessarily have discontinuities in $w_{x}(x, y)$ at every $x=x_{i}$. In essence, the arclength constraint behaves as if it were a boundary condition on $w$ at each $x=x_{i}$, and the overall problem decouples into a collection of regions that must be solved separately and then patched together by enforcing the continuity of $w(x, y)$.

A full analysis of the minimisers of (18) was not achieved during the MISG. The minimisers of (18) having discontinuities in $w_{x}$ suggests that this may not be a good model of a loaded screen. If the minimisation of (18) were a good model of the deformation of a screen, then we would expect to see vertical kinks in the screen along lines connecting the tabbed constraints. While the presence of tabs clearly has an effect on the shape of a loaded 
screen, their impact is not so severe as to lead to the appearance of sharp edges in the middle of the screen.

However, if we isolate one segment from the screen (between two pairs of tabs) and assume periodicity of $w$ while allowing discontinuities in $\boldsymbol{w}_{x}$, then we obtain explicit solutions for the minimisers of an adapted form of (18). That is, we consider the problem of minimising

$$
\begin{array}{r}
E[w(x, y), \lambda]=\int_{0}^{H} \int_{0}^{x} \frac{T_{x}}{2 H}\left(\frac{\partial w(x, y)}{\partial x}\right)^{2}-f(x, y) w(x, y) d x d y \\
+\lambda\left[\int_{0}^{H} \frac{1}{2}\left(\frac{\partial w(0, y)}{\partial y}\right)^{2} d y-\kappa\right],
\end{array}
$$

subject to

$$
w(0, y)=w(X, y), \quad w(0,0)=w(0, \mathrm{H})=w(X, 0)=w(X, \mathrm{H})=0,
$$

where $\mathrm{X}$ represents the width of a single segment (i.e., the distance between pairs of tabs).

Then, we take a functional derivative as before to obtain

$$
\begin{aligned}
\left.\frac{\partial E}{\partial w}\right|_{\eta}=\int_{0}^{H} \int_{0}^{x} \frac{T_{x}}{H} \frac{\partial w(x, y)}{\partial x} \frac{\partial \eta(x, y)}{\partial x}- & f(x, y) \eta(x, y) d x d y \\
& +\lambda \int_{0}^{H} \frac{\partial w(0, y)}{\partial y} \frac{\partial \eta(0, y)}{\partial y} d y .
\end{aligned}
$$

Integrating by parts and exploiting the boundary conditions then gives

$$
\begin{aligned}
\left.\frac{\partial E}{\partial w}\right|_{\eta}=\int_{0}^{H} \int_{0}^{x}[ & \left.-\frac{T_{x}}{H} \frac{\partial^{2} w(x, y)}{\partial x^{2}}-f(x, y)\right] \eta(x, y) d A \\
& +\int_{0}^{H}\left[-\frac{T_{x}}{H}\left[\frac{\partial w(0, y)}{\partial x}\right]^{ \pm}-\lambda \frac{\partial^{2} w(0, y)}{\partial y^{2}}\right] \eta(0, y) d y
\end{aligned}
$$


where

$$
\left[\frac{\partial w(0, y)}{\partial x}\right]^{ \pm}=\frac{\partial w\left(0^{+}, y\right)}{\partial x}-\frac{\partial w\left(\mathrm{H}^{-}, y\right)}{\partial x}
$$

This can now be solved. At a minimiser of E, (25) must be zero for all test functions $\eta(x, y)$, including choices of $\eta(x, y)$ where $\eta(0, y) \equiv 0$. Hence, $w(x, y)$ must solve

$$
-\frac{T_{x}}{H} \frac{\partial^{2} w(x, y)}{\partial x^{2}}=f(x, y)
$$

and, assuming that $f$ is constant and enforcing the periodicity constraint in $w$, we find that

$$
w(x, y)=\frac{f H}{2 T_{x}} x(X-x)+Q(y)
$$

Now, by considering general choices of $\eta(x, y)$ where $\eta(0, y) \not \equiv 0$, we find that $\mathrm{Q}(\mathrm{y})$ must obey

$$
Q^{\prime \prime}(y)=-\frac{f X}{\lambda}
$$

and that $Q(y)$ must also satisfy the length constraint associated with $\frac{\partial E}{\partial \lambda}=0$, which takes the form

$$
\frac{1}{2} \int_{0}^{\mathrm{H}} \mathrm{Q}^{\prime}(\mathrm{y})^{2} \mathrm{dy}=\kappa .
$$

Applying the boundary conditions $\mathrm{Q}(0)=\mathrm{Q}(\mathrm{H})=0$, we therefore find that

$$
Q(y)=\frac{\sqrt{6 \kappa}}{H^{3 / 2}} y(H-y)
$$

and hence the overall solution for $w(x, y)$ is

$$
w(x, y)=\frac{f H}{2 T_{x}} x(X-x)+\frac{\sqrt{6 \kappa}}{H^{3 / 2}} y(H-y)
$$

and the force on the tabs is

$$
\lambda=\frac{f X H^{3 / 2}}{2 \sqrt{6 \kappa}} .
$$


Figure 15: A minimiser of (23) as given in (33). Note that $\boldsymbol{w}(0, \mathbf{y})=\boldsymbol{w}(\mathbf{W}, \mathbf{y})$, but the derivative of $w$ would undergo an abrupt change if we were to show the periodic extension of $w$. In this simulation, $f=6.2 \mathrm{~Pa}$, corresponding to a breeze of $10 \mathrm{~km} \mathrm{~h}^{-1}$ using Bernoulli's law, and the corresponding force on the tabs is $14 \mathrm{~N}$.

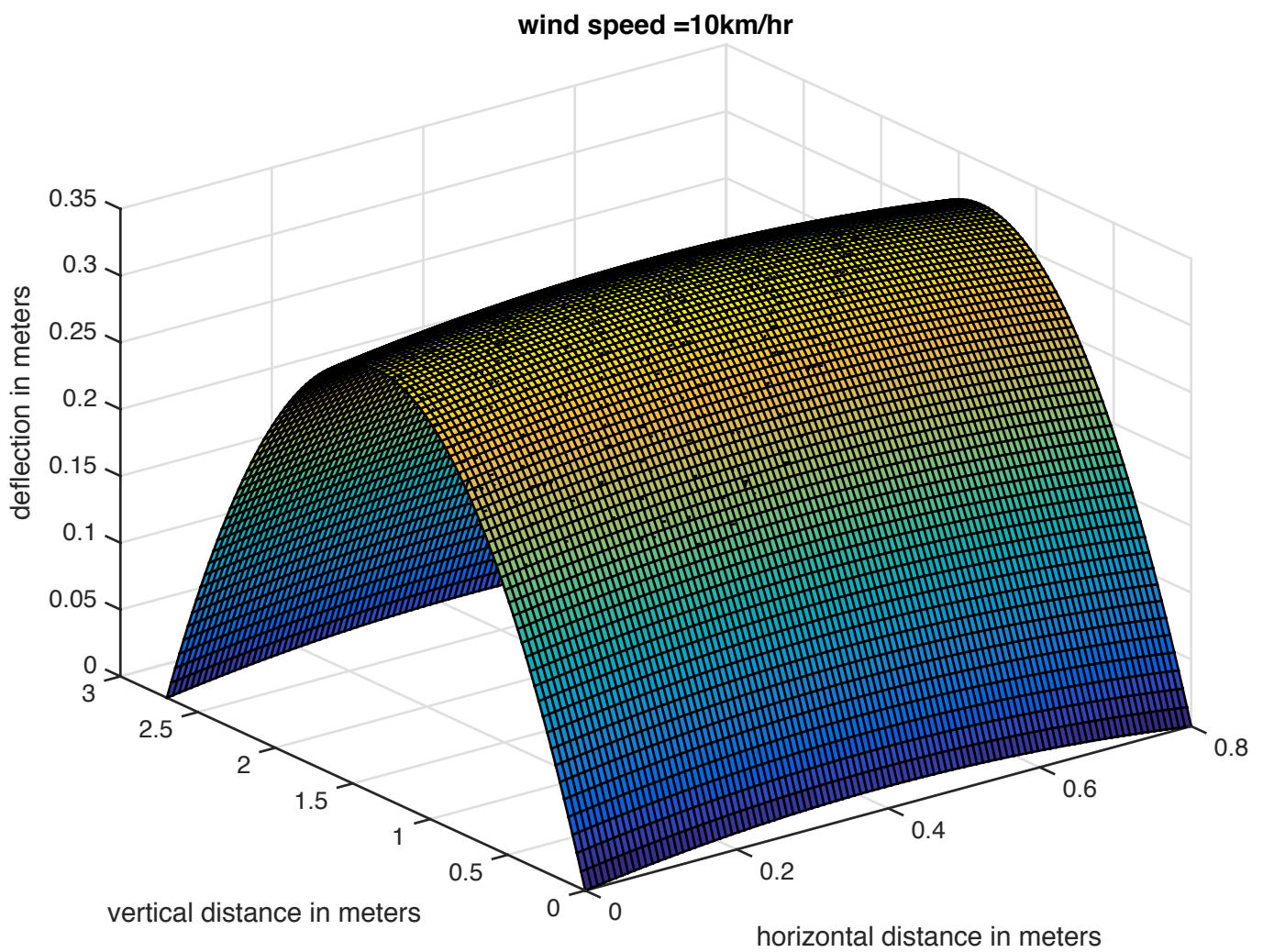


An illustration of the function $w(x, y)$ from (33) is shown in Figure 15. Based on the assumption of six pairs of evenly spaced tabs, we use $X=0.8 \mathrm{~m}$, and based on the assumption that the allowed 'give' in each tab is around $5 \mathrm{~cm}$ (for a total of $10 \mathrm{~cm}$ in each pair of tabs), we use $\mathrm{k}=0.1 \mathrm{~m}$. Taking $\mathrm{H}=2.7 \mathrm{~m}$, $\mathrm{T}_{\mathrm{x}}=60 \mathrm{~N}$, and $\mathrm{f}=6.2 \mathrm{~Pa}$ (corresponding to a breeze of $10 \mathrm{~km} \mathrm{~h}^{-1}$ or $2.8 \mathrm{~m} \mathrm{~s}^{-1}$ by Bernoulli's law), we obtain the results shown, and we find that $\lambda=14 \mathrm{~N}$, indicating that there is only a very small load on the tabs compared to their critical load of $350 \mathrm{~N}$.

Although the force on the tabs is very small, we still observe that the deformation of the screen in Figure 15 is quite large, reaching a maximum deflection of more than $30 \mathrm{~cm}$. This seems unrealistic for such a low load, and it would appear that further work is needed on the membrane models in this section. However, as (23) has an explicit solution for the minimiser, we make some general statements about the scaling behaviour of the system. Equation (34) suggests that the force on the tabs is independent of the horizontal tension in the screen, but is proportional to the load on the screen and inversely proportional to the square root of the amount of 'give' in the system. While it makes sense that the force on the tabs should increase with the pressure on the screen and decrease with the amount of 'give' in the system, it seems surprising that there is complete decoupling between the horizontal problem (which involves the horizontal tension) and the vertical problem (which gives the force on the tabs). We suspect that this is explained by the fact that we simplified our equations by assuming that the solution is periodic. This means that our model screen does not satisfy boundary conditions $w=0$ on its left and right sides.

Further analysis is necessary in order to determine whether the model presented in this section could be modified in order to deal with some of its disadvantages (such as the kinked profile that it predicts for the screen deformation), and to determine whether the prediction that the load on the tabs is independent of the horizontal tension is a valid conclusion. 


\subsection{Membrane models with tension modulation}

\subsubsection{Tension modulation in a strand}

In order to develop models of a membrane with tension modulation, it is useful to start by considering a string with tension modulation. Indeed, from an energetic viewpoint, this is a very natural way to analyse the problem of the meshwork screens developed by Centor. The total elastic energy of the screen is the sum of the elastic energy along the horizontal strands and the elastic energy along the vertical strands.

For a strand fixed at $x=0$ and $x=L$ and deformed to follow a curve $z=w(x)$ where $w(0)=w(\mathrm{~L})=0$, the elastic energy associated with the deformation is

$$
E_{\text {elast }}=\int_{0}^{L} \frac{T(x) \varepsilon(x)}{2} d x-E_{0},
$$

where $T$ is the tension, $\varepsilon$ is the strain, and $E_{0}$ is the elastic energy associated with the undeformed strand stretching from 0 to L. For consistency, strain is measured in terms of the difference between the deformed length and the stress-free length, all divided by $\mathrm{L}$.

While we expect that $w(x)=0$ is an equilibrium in the absence of external forces, this does not necessarily represent the stress-free state if there is pre-existing tension in the strand (i.e., if the strand was stretched from its stress-free state in order to stretch from 0 to L). Let the strand obey Hooke's law, and let the spring constant of the strand be $k$, so that $T=k \varepsilon$. Then, if the unstretched length of the strand is $\mathrm{L}_{0}$, we find that after the strand is stretched to be length $\mathrm{L}$ the tension

$$
\mathrm{T}_{0}=\frac{\mathrm{k}\left(\mathrm{L}-\mathrm{L}_{0}\right)}{\mathrm{L}},
$$

and thus the elastic energy associated with the undeformed strand is

$$
\mathrm{E}_{0}=\frac{\mathrm{k}\left(\mathrm{L}-\mathrm{L}_{0}\right)^{2}}{2 \mathrm{~L}} \text {. }
$$


Now, let the stretched strand be subject to a perpendicular load so that it deforms from its initially straight shape. Then, we define the strain in terms of the local arclength, so that

$$
\mathcal{E}(x)=\sqrt{1+\left(\frac{d w}{d x}\right)^{2}}-\frac{L_{0}}{L} .
$$

If the deflection is small, so that $\frac{\mathrm{d} w}{\mathrm{~d} x} \ll 1$, we then find

$$
\varepsilon(x)=\frac{1}{2}\left(\frac{d w}{d x}\right)^{2}+\frac{L-L_{0}}{L},
$$

a fibre or equivalently,

$$
\varepsilon(x)=\frac{1}{2}\left(\frac{d w}{d x}\right)^{2}+\frac{T_{0}}{k} .
$$

Noting that $T(x)=k \varepsilon(x)$, substituting into (35) then yields

$$
E_{\text {elast }}=\int_{0}^{L} \frac{T_{0}}{2}\left(\frac{d w}{d x}\right)^{2}+\frac{k}{8}\left(\frac{d w}{d x}\right)^{4} d x .
$$

The first term is identical to the conventional energy associated with deforming a strand under tension. The second term is more unusual, and can be thought of as being a tension modulation term that represents the effect of stretching on the tension in the string. If the initial tension is high or the deflection is small, so that $T_{0} \gg k\left(w_{x}\right)^{2}$, then the tension modulation term can be ignored. However, if $T_{0}$ is very small, then the tension modulation term has the potential to be important.

Now imagine a strand under a perpendicular load, $f(x)$. Based on the elastic energy in (41), finding the displacement profile $w(x)$ of a loaded strand is equivalent to finding a minimiser of

$$
E=\int_{0}^{L} \frac{T_{0}}{2}\left(\frac{d w}{d x}\right)^{2}+\frac{k}{8}\left(\frac{d w}{d x}\right)^{4}-f(x) w(x) d x
$$


subject to the constraints $w(0)=w(\mathrm{~L})=0$.

The Euler-Lagrange equation associated with this minimisation problem is

$$
-T_{0} \frac{d^{2} w}{d x^{2}}-\frac{3 k}{2}\left(\frac{d w}{d x}\right)^{2} \frac{d^{2} w}{d x^{2}}=f(x) .
$$

In contrast, the statically-loaded equivalent of the Kirchhoff-Carrier equation (used in models of vibrations with tension modulation $[2,3,15]$ ) takes the form

$$
-\left\{T_{0}+\frac{k}{2 L} \int_{0}^{L}\left[w^{\prime}(\bar{x})\right]^{2} d \bar{x}\right\} \frac{d^{2} w}{d x^{2}}=f(x) .
$$

While Bank [3] considered an energy-based argument for the Kirchhoff-Carrier equation as applied to a vibrating string, it is very difficult to phrase the static Kirchhoff-Carrier equation as an energy minimisation. However, for developing finite element simulations it is necessary to phrase problems as energy minimisations; as a result, we use (41) as the basis for our model of a membrane with tension modulation rather than the Kirchhoff-Carrier equation.

\subsubsection{Critical loads for an untabbed screen}

To model the 'original' Centor design for screen doors (i.e., without tabs), the Kirchhoff-Carrier equation is applicable. To model screens with tabs, the problem is inherently two-dimensional and so it is very difficult to make progress without using numerical simulations; in this case, it is a significant disadvantage that the Kirchhoff-Carrier equation is not well-suited to finite element analysis. However, in the absence of tabs, the vertical strands essentially play no role in determining the shape of the deformed screen, and it is straight forward to use the 1D Kirchhoff-Carrier equation to analyse the deformation of the screen as a function of horizontal position. 
To achieve this, we first rewrite (44) as a stress balance per unit length in the vertical direction, so that

$$
-\left\{\frac{T_{0}}{H}+\frac{k \rho_{y}}{2 W} \int_{0}^{W}\left[w^{\prime}(\bar{x})\right]^{2} d \bar{x}\right\} \frac{d^{2} w}{d x^{2}}=f(x) .
$$

where $T_{0}$ is the horizontal tension applied to the screen $(\mathrm{N}), \mathrm{H}$ and $\mathrm{W}$ are the height of the width of the screen respectively $(\mathrm{m}), \mathrm{k}$ is the spring constant of an individual strand $(\mathrm{N}), \rho_{y}$ is the density of strands in the vertical direction $\left(\mathrm{m}^{-1}\right)$, and $f(x)$ is the pressure applied $\left(\mathrm{N} \mathrm{m}^{-2}\right)$ perpendicular to the screen.

Consider the case where the load, $f(x)$, is constant, and define

$$
\alpha=\int_{0}^{W}\left[w^{\prime}(\bar{x})\right]^{2} \mathrm{~d} \bar{x} .
$$

Solving (45) subject to the boundary conditions $w(0)=w(L)=0$, we find

$$
w(x)=\frac{f H W}{k \alpha \rho_{y} H+2 T_{0} W} x(W-x) .
$$

Substituting into (46) and rearranging then yields a cubic equation in terms of $\alpha$ :

$$
\alpha^{3}+\frac{4 T_{0} W}{k \rho_{y} H} \alpha^{2}+\frac{4 T_{0}^{2} W^{2}}{k^{2} \rho_{y}{ }^{2} H^{2}} \alpha-\frac{f^{2} W^{5}}{3 k^{2} \rho_{y}^{2}}=0 .
$$

For positive values of the parameters, this has one real solution, which is also positive.

Once $\alpha$ is determined by solving the cubic, it is possible to substitute back into (47) to obtain a solution for $w(x)$. Importantly, the maximum deformation of the screen is

$$
w_{\max }=\frac{f H W^{3}}{4 k \alpha \rho_{y} H+8 T_{0} W},
$$

and the total horizontal force on the springs $(\mathrm{N})$ is

$$
\mathrm{T}_{\text {total }}=\mathrm{T}_{0}+\frac{k \rho_{\mathrm{y}} \alpha \mathrm{H}}{2 \mathrm{~W}} .
$$


This last term, $T_{\text {total }}$, is of particular importance, since it is the high horizontal tension that leads to the failure of the screen in the case of the original (nontabbed) screen design. If the wind is sufficiently strong that this additional tension is much greater than the original tension in the system, then we can neglect $\mathrm{T}_{0}$ in (48) and obtain an equation that is much easier to solve. This yields

$$
\alpha=\frac{f^{2 / 3} W^{5 / 3}}{3^{1 / 3} k^{2 / 3} \rho_{y}^{2 / 3}},
$$

and hence

$$
\mathrm{T}=\frac{\mathrm{f}^{2 / 3} \mathrm{k}^{1 / 3} \rho_{\mathrm{y}}{ }^{1 / 3} \mathrm{HW}^{2 / 3}}{2 \cdot 3^{1 / 3}}
$$

Now, the parameters $k, \rho_{y}, H$ and $W$ are all known, and the pressure $f$ increases as the square of wind speed (as discussed in Section 3). Hence, equation (52) predicts that the additional load on the springs holding the screen in place will increase with the $4 / 3$ power of wind speed. Moreover, the procedure described above can be used to predict the additional load on the springs holding the screen in place in response to wind loading.

As discussed in Section 2, experimental observations give the result that the critical value of $\mathrm{T}$ for failure of springs is $500 \mathrm{~N}$, while $\mathrm{k}=200 \mathrm{~N}, \rho_{\mathrm{y}}=600 \mathrm{~m}^{-1}$, $\mathrm{H}=3 \mathrm{~m}$ and $\mathrm{W}=5 \mathrm{~m}$. Using (52) to solve for $\mathrm{f}$ so that $\mathrm{T}=500 \mathrm{~N}$, this gives the result that the critical pressure for failure is around $6 \mathrm{~Pa}$. Assuming the classical Bernoulli's law to relate pressure to wind speed (i.e., without introducing the pressure coefficients described in Section 3), this corresponds to a wind speed of only $3.2 \mathrm{~m} \mathrm{~s}^{-1}$, which is only a light breeze. While the untabbed screens were known to fail at low wind speeds, this seems unrealistically low.

Figure 16 shows the results of including a nonzero $\mathrm{T}_{0}$ of $120 \mathrm{~N}$ and calculating $\alpha$ based on (48). This reduces the critical pressure even further, bringing it as low as 4.4 Pa. While this is clearly unrealistic, it does emphasise that the untabbed screens are much weaker than the tabbed screens analysed in other models. 
Figure 16: The horizontal tension on a $3 \mathrm{~m}$ by $5 \mathrm{~m}$ screen, based on the Kirchhoff-Carrier model in equation (45). Based on the properties of the screens described in Section 2, we use $T_{0}=120 \mathrm{~N}, \mathrm{k}=200 \mathrm{~N}, \rho_{\mathrm{y}}=600 \mathrm{~m}^{-1}$, $\mathrm{H}=3 \mathrm{~m}$ and $\mathrm{W}=5 \mathrm{~m}$. We find that the uniform pressure corresponding to failure of the springs is approximately $4.4 \mathrm{~Pa}$.

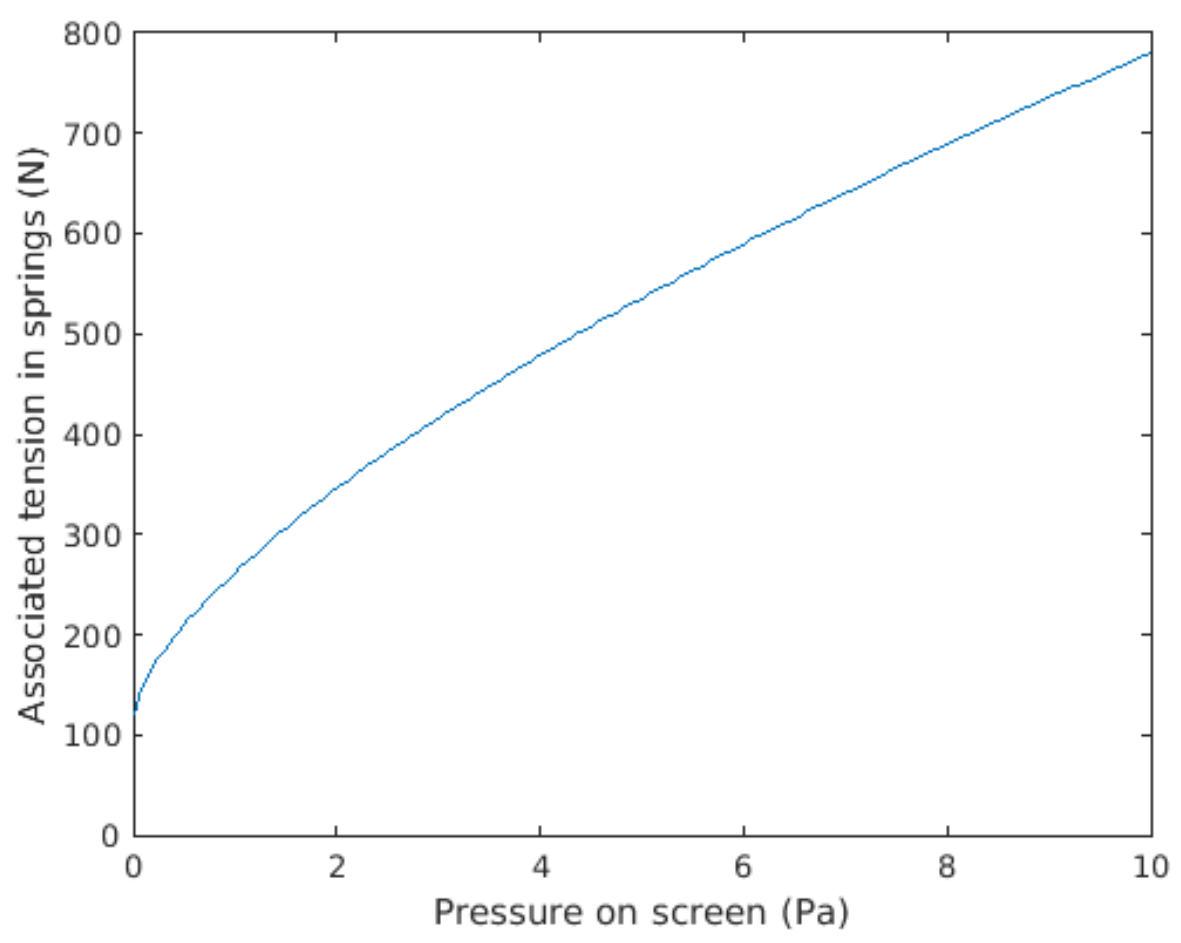


There are a number of reasons why the analysis in this section might lead to very low critical pressures. For example, the model developed in this section does not take into account elastic or geometric nonlinearities, which are likely to be important at the large deformations observed in untabbed screens. Moreover, we converted between pressure and wind velocity without accounting for the porosity of the screen or for any release of pressure associated with the wind escaping around the top and bottom edges. In the case of an untabbed screen, this is likely to lead to a significantly reduced pressure for a given wind speed compared to what would be predicted using Bernoulli's law.

With the caveat that the precise values obtained for the critical pressure are likely to be underestimates, this model gives a scaling relationship between the physical parameters of the screen, the applied load, and the increased tension on the horizontal supports. Assuming that the pressure due to wind loading scales with the square of wind velocity $v$ and that the initial horizontal tension is small enough to be neglected, we find that

$$
\mathrm{T} \propto v^{4 / 3} k^{1 / 3} \rho_{y}{ }^{1 / 3} H W^{2 / 3} .
$$

If the wind velocities corresponding to the failure of an untabbed screen are known, then this could be used to estimate the wind velocities corresponding to the failure of an equivalent untabbed screen with a different size, or composed of strands with different elastic properties.

\subsubsection{Continuum finite element model with tension modulation}

Now consider a screen composed of strands aligned in the horizontal and vertical directions. All strands have the same spring constant $k(\mathrm{~N})$, but (as noted in Section 2) the density of strands in the horizontal and vertical directions are different. Even before loading, the horizontal strands are held under a tension $T_{x 0}(N)$, while the vertical strands are initially stress-free. As a result, the only contribution to the energy of the vertical strands comes 
from the tension modulation term, while the horizontal strands have both a 'classical' membrane term and a tension modulation term. The horizontal and vertical strands are woven together so that we define a single function, $w(x, y)$, for the displacement of the screen from an initial flat state.

Based on this situation, we use (41) to propose a total energy of the form

$$
\int_{0}^{\mathrm{H}} \int_{0}^{W} \frac{T_{x 0}}{2 \mathrm{H}}\left(\frac{\partial w}{\partial x}\right)^{2}+\frac{k \rho_{y}}{8}\left(\frac{\partial w}{\partial x}\right)^{4}+\frac{k \rho_{x}}{8}\left(\frac{\partial w}{\partial y}\right)^{4}+f(x, y) w(x, y) d x d y
$$

where $\rho_{x}$ is the density of vertical strands (i.e., the density of strands across the width of the screen) $\left(\mathrm{m}^{-1}\right), \rho_{x}$ is the density of horizontal strands $\left(\mathrm{m}^{-1}\right)$, $f(x, y)$ is the applied load on the screen $(\mathrm{Pa})$; and $\mathrm{H}$ and $\mathrm{W}$ are the height and width of the screen respectively $(\mathrm{m})$.

This is well-suited to being solved using finite element methods, with zerodisplacement boundary conditions at the left and right hand ends (and at the tabs on the upper and lower edges). Unlike the tabbed model discussed in Section 5.2, there is no give in the tabs and they are treated as zerodisplacement boundary conditions. However, the screen is still able to deform outwards, because the vertical strands are treated as being elastic rather than inextensible.

Considering a $3 \mathrm{~m}$ by $5 \mathrm{~m}$ screen with six pairs of tabs, we obtain the results given in Figure 17. This shows the displacements of a screen subject to a uniform $80 \mathrm{~Pa}$ pressure, corresponding to a wind speed of around $11.5 \mathrm{~m} \mathrm{~s}^{-1}$ using Bernoulli's law. We find that the middle of the screen bows by about $16 \mathrm{~cm}$, which is consistent with Centor's observations. Values for the model parameters $\left(T_{x 0}, k, \rho_{x}, \rho_{y}, H, W\right)$ are as given in Section 2 and are stated explicitly in the caption of Figure 17.

The advantage of using the method described in this section for determining the deformation of a screen is that it runs quickly and efficiently to obtain reasonable results without having to track the deformation of every individual fibre. The reduction in computational complexity associated with replacing 
Figure 17: Displacement in metres as a function of position (also in metres) in the finite element simulations of the tension modulation model. Parameters used are $\mathrm{f}(\mathrm{x}, \mathrm{y})=80 \mathrm{~Pa}, \mathrm{~T}_{\mathrm{x} 0}=60 \mathrm{~N}, \mathrm{k}=200 \mathrm{~N}, \rho_{\mathrm{x}}=670 \mathrm{~m}^{-1}, \rho_{y}=510 \mathrm{~m}^{-1}$, $\mathrm{H}=3 \mathrm{~m}, \mathrm{~W}=5 \mathrm{~m}$. Tabs are each $0.05 \mathrm{~m}$ long, and are treated as zerodisplacement boundary conditions that are evenly spaced along the top and bottom edges of the domain. The effects of gravity and other forces are ignored.

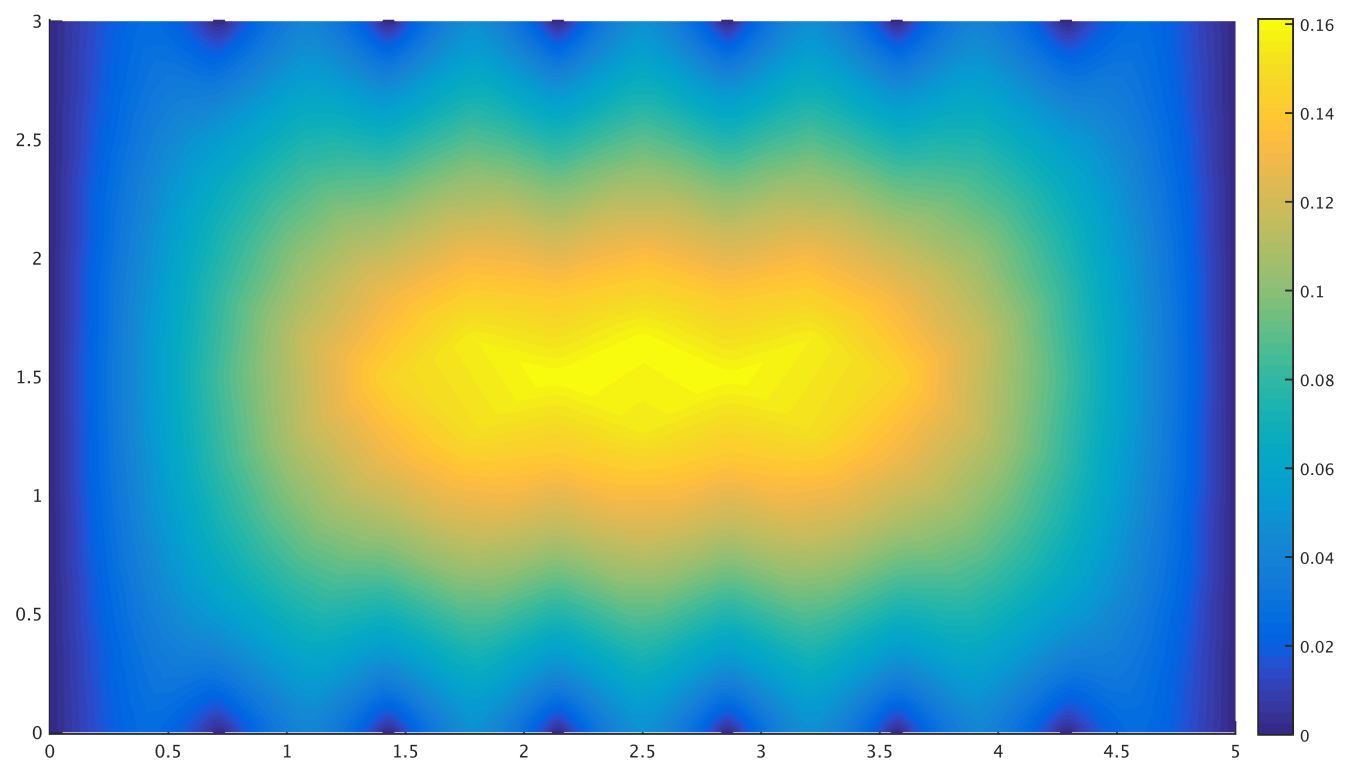


the fibrous structure of the screen with a continuum gives this method a significant advantage over the mass-and-spring model described in Section 4, or the fibre-based FEM model described in Section 6. Moreover, it is not necessary to introduce 'flexion springs' or other elements in order to obtain realistic results: the plain membrane equation with tension modulation can be used to obtain a reasonable picture of the deflection of a screen.

However, this method also has some disadvantages compared to the other methods explored. During the MISG, we did not evaluate the loads on the tabs or on the left and right hand sides of the screen associated with the model. While these results could be obtained, they are not as straightforward to evaluate as they are using the methods described in Section 4. Further work is needed in order to obtain useful load data from the tension modulation model. Additionally, there is still a question around whether (54) is an appropriate model for a screen with tension modulation: while Section 5.3.1 justified it from the equations of elasticity for individual strands, the equations that we use are not the same as those in the classical literature on tension modulation for membranes [2], and (54) may not provide an accurate picture of the energy of a screen under tension modulation.

\subsection{Membrane models with geometric nonlinearity}

Another way of developing a membrane model for screen deflection is to use the cornea model developed by Okrasiński and Płociniczak [10] as a theoretical basis. The underlying physical model is that of a homogeneous membrane clamped at the boundaries, which is then put under load with a uniform pressure perpendicular to its plane at rest. The model assumes a circular domain in which the PDE is axisymmetric, which allows a reduction to 1D. We extend the model to a $2 \mathrm{D}$ rectangular domain $\mathrm{D}$ under the assumption that the tension may still be approximated by a scalar constant throughout. 
The modified differential equation is

$$
-T \nabla^{2} h(x, y)+k h(x, y)=\frac{P}{\sqrt{1+|\nabla h(x, y)|^{2}}},
$$

where $T$ is the tension, $h(x, y)$ is the deflection at the point $(x, y), k$ is elasticity (constant), $\mathrm{P}$ is pressure, and $\left.\mathrm{h}\right|_{\partial \mathrm{D}}=0$. The presence of the term on the denominator of the right hand side of $(55)$ accounts for the geometric nonlinearity of the deforming membrane.

The corneal model does not take into account permeability of the membrane to wind. It also assumes that the material is isotropic. From observation, the permeability is highly nonlinear with respect to wind speed. For low speeds $\left(0.5 \mathrm{~m} \mathrm{~s}^{-1}\right)$, the permeability is effectively zero. We expect the permeability to tend asymptotically to a constant for increasing wind speeds. Furthermore, this model neglects gravity, assuming that once wind speed is sufficiently high the difference in tension between top and bottom attachment points due to gravity is small compared to the tension induced by wind pressure.

Unfortunately time constraints meant tension and distributed force data were not calculated for this model. However, preliminary results are presented along with experimental output in Section 7.

\section{Finite element models of a fibrous screen}

Another way of modelling the deformation of a screen under load is to model the deformations of the strands using a finite element package such as COMSOL. This approach bears some important similarities with the mass-spring model, since it involves working with the elastic properties of individual strands rather than the averaged, continuum behaviour of the screen. However, unlike the mass-spring model, this approach means that each strand is treated as a long rod, rather than a sequence of springs, and the mass is evenly distributed 
along each rod rather than being localised to point masses at the junctions. This means there is no need to introduce 'flexion springs' and 'shear springs' in order to obtain reasonable results, and the finite element model has the potential to be more realistic than the mass-spring model.

However, there are disadvantages to using a finite element model that tracks the deformation of each strand independently. The first difficulty is associated with developing an appropriate initial condition for the model. When a screen is made, the initially-straight strands are woven together and stretched, heat is then applied to allow the plastic coatings of the strands to coalesce, forming the junctions of the screen. It is difficult to account for these 'weak' junctions in a strand-based finite element model, and it is even more difficult to construct a 'woven' initial condition, where each strand goes alternately above and below the perpendicular strands.

As a proof of concept that finite element packages can be used to model fibrous screens, we developed a finite element model for a small $(12 \mathrm{~cm}$ by $20 \mathrm{~cm})$ section of screen, constructed from equally spaced warp and weft strands as shown in Figure 18. In order to deal with the difficulty of constructing a woven initial condition, we treat the horizontal strands as being straight, parallel rods, and the vertical strands are alternately laid above and below the horizontal strands. This leads to the basic repeating unit shown on the left hand side of Figure 18, and the overall structure shown on the right hand side of Figure 18.

For the finite element model, it is necessary to know the density, Young's modulus, and Poisson's ratio of the material from the which the yarns are made. The material density is given by the manufacturers as around $1000 \mathrm{~kg} \mathrm{~m}^{-3}$; the Young's modulus is estimated by dividing the spring constant, $k=200 \mathrm{~N}$, by the cross-sectional area of an individual strand; and the Poisson's ratio for a typical plastic is around 0.4. In the simulations that follow, we use Young's modulus of $6.2 \times 10^{10} \mathrm{~Pa}$. This is inaccurate because of a mistake in calculations during the MISG: a more realistic value of the Young's modulus based on a spring constant of $200 \mathrm{~N}$ would be $7.1 \times 10^{8} \mathrm{~Pa}$. However, since 
Figure 18: The structure of the screen for finite element simulations. For simplicity, we use a square mesh of strands rather than the rectangular mesh discussed in Section 2, and the strands are lain on top of each other rather than woven together.
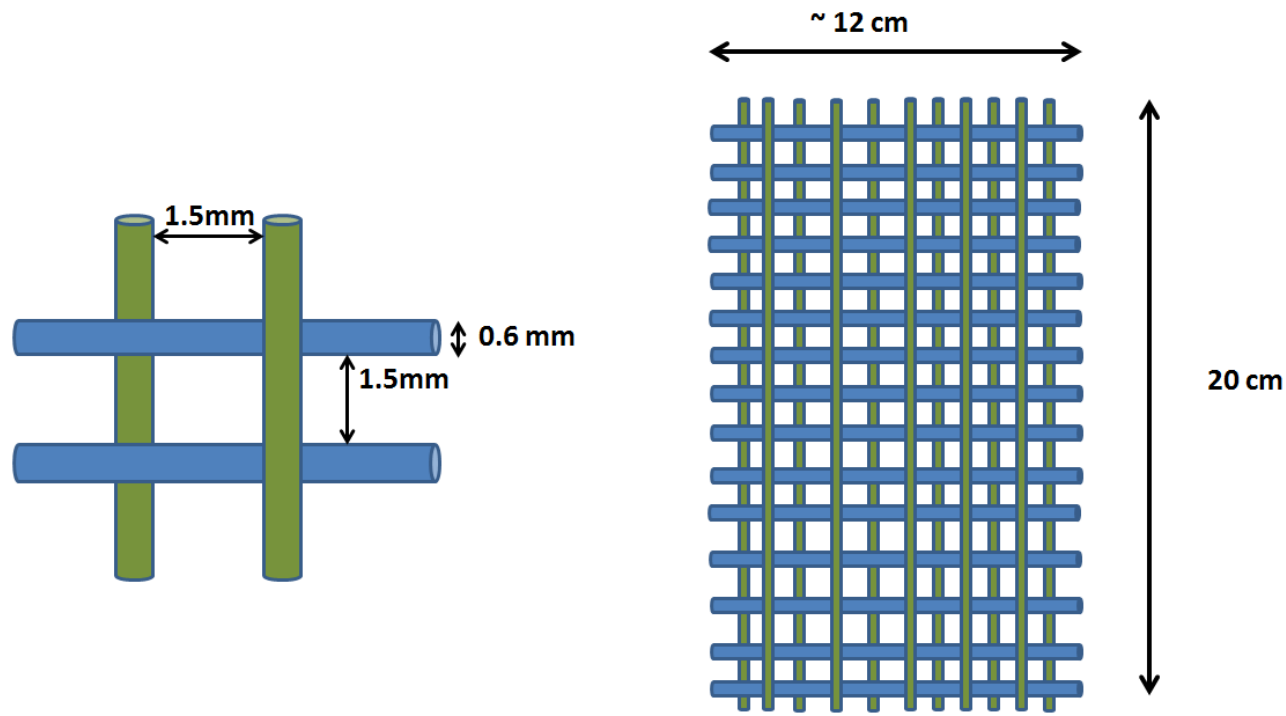

the finite element model developed here is mainly used as a proof of concept, we have not corrected these results.

As a demonstration that it is possible to mix free and fixed boundary conditions (as would be necessary for a model including both fixed horizontal boundaries and tabs on the vertical boundaries), we consider the boundary conditions shown in Figure 19. Then, applying a distributed load of $2 \mathrm{~N}$ across the sample of screen material leads to the deformation depicted in Figure 20, obtained using COMSOL. Since the sample is very small and the Young's modulus is large, we only obtain very small displacements (at most $0.4 \mathrm{~mm}$ ), but the methods described in this section could be adapted to describe larger 
Figure 19: Boundary conditions used to generate the results in this section, demonstrating that it is possible to apply both fixed and free boundary conditions using a finite element package.

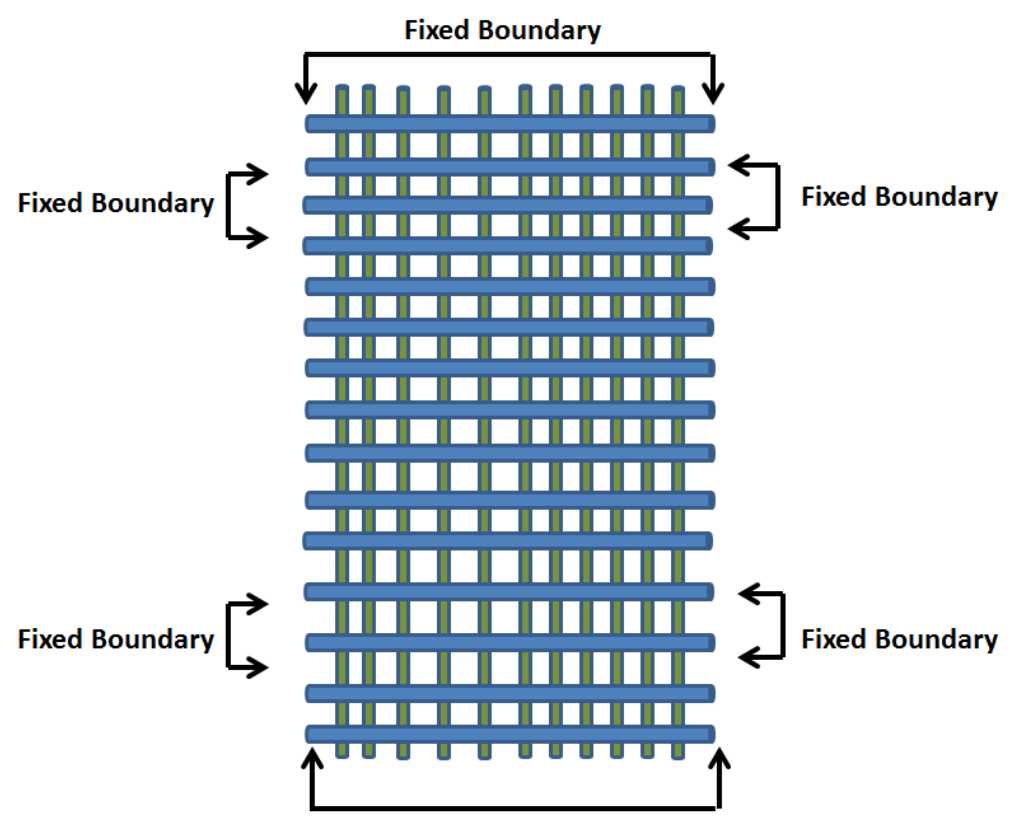

Fixed Boundary

screens. The only limitations are those described above: the difficulty in constructing an appropriate initial condition, and the computational power required to solve for the internal deformations of every strand in the screens.

\section{Validation Experiment}

As a way of understanding how the screen deformed under wind load, a simple experiment was set up in the laboratory to blow air onto an actual $3 \mathrm{~m}$ 
Figure 20: The deformation of a small screen segment under a $2 \mathrm{~N}$ load, where all of the strands in the screen are modelled as elastic rods using the COMSOL computational package. The maximum displacement is around $0.4 \mathrm{~mm}$.
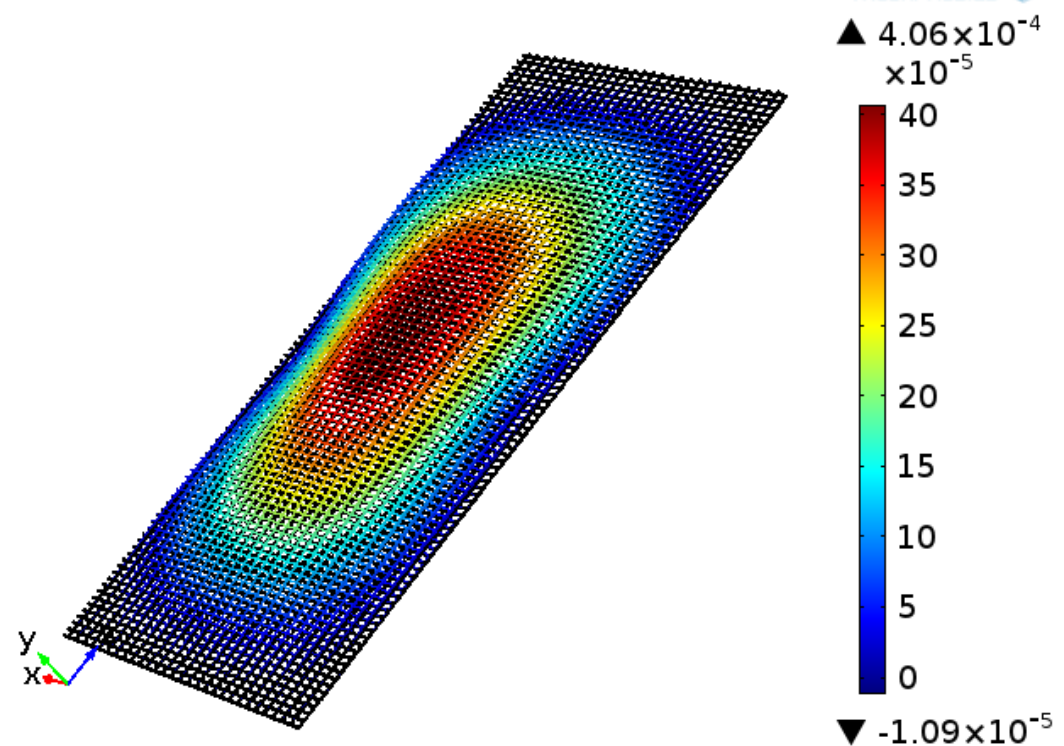

by $5 \mathrm{~m}$ screen door and measure its deflected shape. The aim of this was to use the data obtained from the experiments to fit the parameters in (55). Additionally, it was hoped that this would provide an 'order of magnitude' check for deflection measurements coming from the models.

The experimental setup involved three industrial fans, which blew air at a range of wind speeds onto the screen door. All tests were performed with the airflow perpendicular to the door. Deflection readings were obtained for seven seconds (200 frames) using a Microsoft $\AA$ Kinect $\AA$ sensor, and wind speeds in front and behind the screen were measured using an anemometer (Kestrel 4500 Weather Meter). The experimental setup is shown in Figure 21.

We performed seven trials with different average wind speeds, namely $0.0,0.5$, 
Figure 21: Laboratory experimental test setup.

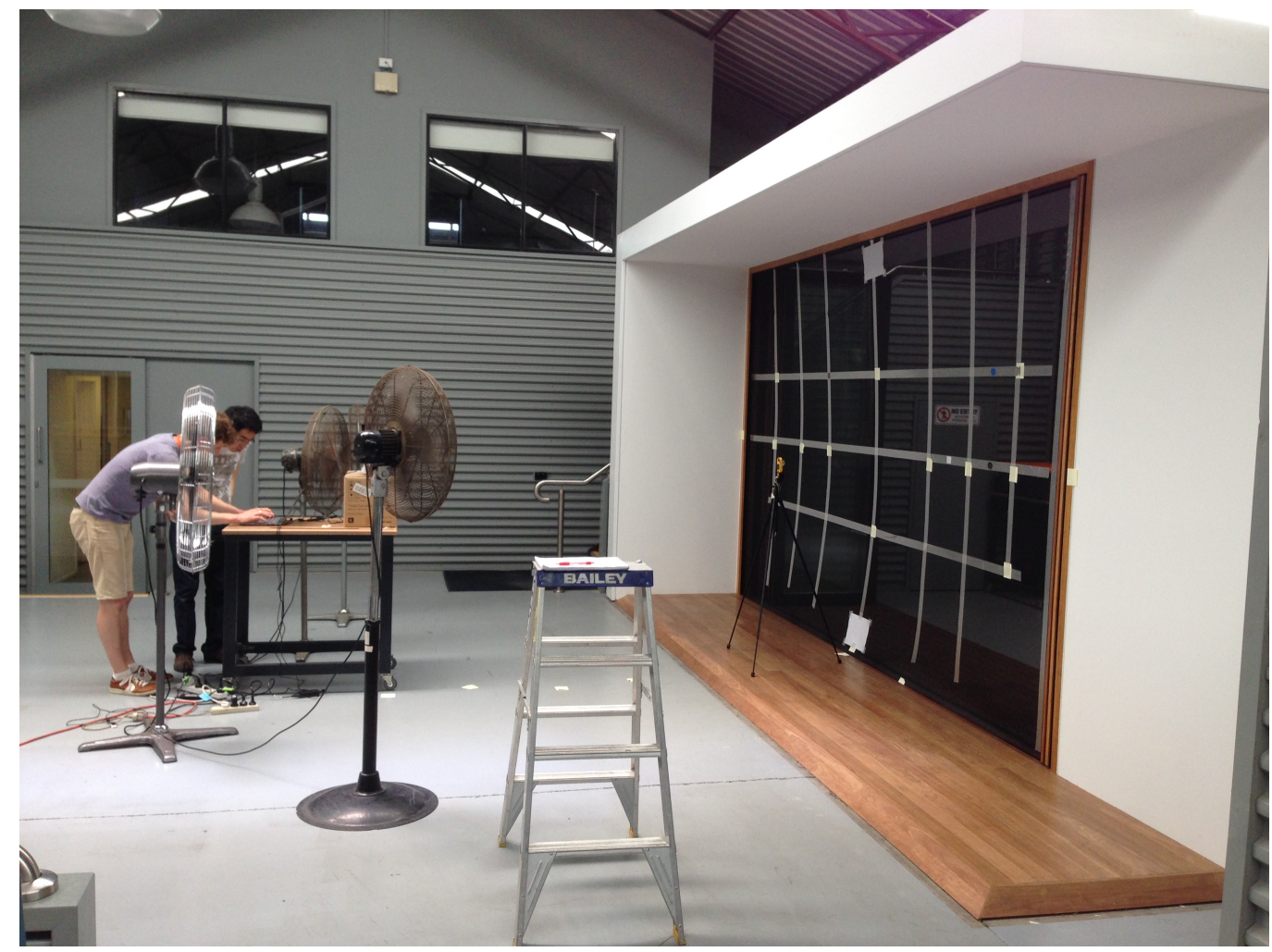

1.0, 1.5, 2.3, 4.5, $6.0 \mathrm{~m} \mathrm{~s}^{-1}$. However, from Figure 21, the fan arrangement was unable to apply a uniform load over the entire door. As such the speeds themselves are not representative of actual incident wind speeds, as used in the mathematical models. Irrespective, the screen deflected in a manner similar to what was expected. Any influence of uneven loading on the deflected shape appeared to disappear for the larger wind speeds. Wind speeds behind the screen quickly saturated at about $1 \mathrm{~m} \mathrm{~s}^{-1}$ with a frontal wind speed of about $1.5 \mathrm{~m} \mathrm{~s}^{-1}$. This is due to the presence of a wall relatively close behind the screen, which meant the wind could not flow freely through the screen, similar to what would be expected to occur with a door on a building. This 
appears to qualitatively confirm the findings of Robertson et al. [14], with regards to porous windward walls behaving similarly to impermeable walls when located on a building.

Figure 22 shows an example of the deflected shape measured for an individual experiment with an applied average wind speed of $1.5 \mathrm{~m} \mathrm{~s}^{-1}$. Red lines show smoothed displacement measurements taken to the white tape grid shown in Figure 21. In this instance the maximum deflection is approximately $100 \mathrm{~mm}$ at the centre of the screen, which is notably less than predicted by each of the models at similar wind speeds.

More detailed experimental modelling of this type could be used to validate and help improve each of the models and their variables. One such attempt was undertaken during the MISG with promising results. The measured deformation shapes from experiments were used to fit the constants in equation (55) using a mean absolute error minimisation technique. Using this approach, we found that we could fit the experimental data to within millimetres (as shown in Figure 22). However, in order to obtain this quality of fit it was necessary to use pressures $\mathrm{P}$ that were different from those that would be obtained from the applied wind velocity using Bernoulli's law. Further optimisation work such as this should be undertaken in tandem with experimental tests.

\section{Discussion}

Considering the models presented in Sections 4 to 6, it is evident that there are many ways to estimate both the deflected shape of the screen door and the load distributed to each of the supporting tabs. Of those discussed, the mass-spring model (described in Section 4) and the membrane models without tension modulation (Section 5.2) both provide a means to estimate the maximum load distributed to the tabs; albeit with caveats. As discussed in those sections, both models appear to substantially overestimate deflections, especially given the experimental results in Section 5.4. This suggests that 
Figure 22: Experimental results obtained for an applied wind speed of $1.5 \mathrm{~m} \mathrm{~s}^{-1}$ (shown as red lines), superimposed with a solution to equation (55) with parameters chosen to minimise the mean absolute error.

\section{MAE: $0.009725[\mathrm{~m}]$}

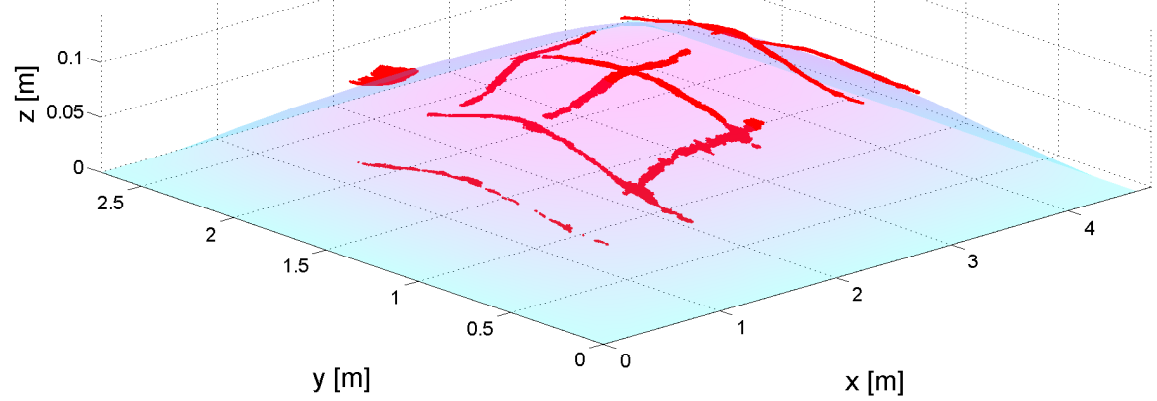

some level of calibration or modification is required before these models could be used in practice, and some skepticism should be applied to the results for the loads experienced by the tabs.

While keeping the points above in mind, it is still considered instructive to explore the application of equation (3) to investigate what positioning the door on a building does to the equivalent wind speed felt by that door. This is done for hypothetical Scenarios 1 and 2 described in Section 3 and the output of the mass-spring model (Section 4).

Applying equation (2) to the wind speeds used in each of the models, equivalent wind speeds are determined that relate model results to pressure loading on the door once it is positioned on a building. For Scenario 1, where the building is considered to be on flat ground, the Mass-Spring model (with some extrapolation of results presented in Table 1) estimates the tab failure threshold of $350 \mathrm{~N}$ is exceeded with a mean wind speed of approximately $30 \mathrm{~m} / \mathrm{s}$. Intuitively this is high. Investigating why this may be the case it appears that 
as wind speed increases, considerably less of the applied dynamic wind force is transferred into the tabs and vertical supports. For example, summing all forces for the $120 \mathrm{~km} / \mathrm{h}$ case (Table 1 ) indicates that only around 45 percent of the applied load is taken by the supports. The large deflections shown in Figure 13 suggests that large proportions of the input energy are being consumed by spring stretching, but this requires further research.

Considering Scenario 2, where the building is considered to be sitting on a hill, mean failure wind speeds are estimated to be $21 \mathrm{~m} / \mathrm{s}$. This represents approximately a 30 percent reduction in wind speed compared with Scenario 1, indicating the importance of surrounding terrain on the resultant wind loads felt on a building (and its doors). This type of hypothetical analysis could be carried out for any number of possible building set ups and could be tailored to the geometric set up of buildings where door failures had been noted in an attempt to validate underlying assumptions used in each of the mechanical models.

Despite possible problems, there are some valuable results obtained from the models developed in Sections 4 to 6. For example, analysis of Table 1 suggests that a maximum of around 4 percent of the total load on a screen is transferred to the individual tabs, which may be a useful result for Centor when estimating the way in which load is transferred to the tabs. Similarly, the results in Section 5.2 suggest that the load on the tabs is proportional to $\mathrm{K}^{-1 / 2}$, where $\mathrm{K}$ is the amount of 'give' in the system before the tabs are weighted. Additionally, the results in Section 5.3.2 suggest that, for an untabbed screen, the load transferred to the side supports increases with the $4 / 3$ power of velocity; if the loads associated with some low wind-speeds were measured, then this would provide a way to extrapolate to determine the wind speeds associated with the failure of an untabbed screen. 


\section{Conclusions}

With the aim of modelling the deflected shape and load redistribution of a large porous screen door subjected to wind load, a load-response modelling framework was developed. Given the complexities of modelling realistic wind loads on a fabric door located on a building, a simplified 'code-based design' methodology was utilised (Section 3) for estimating the load associated with a given wind velocity together with geographical and architectural considerations. This approach allowed wind pressure to be modelled as a time-invariant uniformly distributed load over the entire door and empirical adjustment factors were used to determine equivalent wind speeds based on different conditions. As discussed in Section 8, these could be used to estimate the critical wind speeds associated with the failure of the system, but these results need to be treated with caution.

A mass-spring model (Section 4), several continuum membrane models (Section 5) and an exploratory investigation of a finite element model (Section 6) were developed to simulate the response of the porous door to the applied wind load. The mass-spring model was developed to a point where the loads distributed to the supporting tabs and the deflected shape of the door were estimated for a range of wind speeds. It was found that the maximum load was felt on the central tabs at the top of the door due to the combination of wind load and the mass of the screen itself. Without detailed experimental data to validate results, firm conclusions on the model performance cannot be drawn. However, intuitively it appears that the current model setup generates tab loads that are too low for the applied wind loads and deflections that are too large. We expect that calibration of the spring constants used within the model would improve output deflection and load results.

A membrane model including only small vertical tensions set by arclength constraints between vertically separated tabs was also formulated (Section 5.2). It was found that by imposing this arclength constraint on an infinitely-long, periodic configuration, each segment of the door (i.e., the region between 
tabs) could be analysed to obtain explicit solutions for the deflection and the load on the tabs. As with the mass-spring model, unrealistically large deflections were calculated using this approach. However, based on the set of equations minimised, we hypothesise that load distributed to the tabs is largely independent of horizontal tension within the screen but proportional to the applied wind load and inversely proportional to the square of the 'give' in the system. Further research is required to validate these conclusions.

A membrane model with tension modulation was also considered (Section 5.3.2). Using the Kirchhoff-Carrier equation, a model of the old 'untabbed' door (Figure 1 left) was developed and a scaling relationship was derived that relates maximum tension loads to the wind speed and several of the door parameters. With calibration of the constants of proportionality, this could be a useful tool that would allow prediction of failure wind speeds for screen doors of this type. Preliminary work was undertaken to extend this approach to the new 'tabbed' door, where horizontal and vertical strands required consideration. In the models of 'tabbed' doors with tension modulation, a simple model of tension modulation was introduced in both directions, and the resulting system of equations was solved using finite element methods. Realistic deflections comparable to those reportedly observed in practice were estimated. Unfortunately, load calculations were not carried out during the MISG. We recommend further analysis of this model, given it appears to perform well with regards to deflected shape and magnitude.

A finite element model of a small section of the screen door mesh was simulated in COMSOL and a small wind load applied to assess the ability of this modelling approach to handle multiple boundary conditions and individual strand mesh fabric (Section 6). Numeric results were promising, but long run times and difficult geometric setup were required and thus render the approach impractical for analysis of a full size door.

Finally, preliminary laboratory experiments were carried out on a full size door to estimate the deflected shape under a range of wind loads. Deformations were measured using a Kinect $\AA$ sensor, which measured a maximum deflection 
of approximately $100 \mathrm{~mm}$ for an approach wind speed (not uniform) of up to $7 \mathrm{~ms}^{-1}$. The results obtained match reasonably well to the deflection estimates based on the membrane model with tension modulation, but more experiments are required to validate/calibrate this and each of the other models.

In conclusion, while no definitive solution was found during the MISG timeframe that can provide Centor with estimates of wind speeds expected to cause door failure, several promising models were explored and developed. However, a clear pathway to finding such a solution was provided and the the relative strengths and weaknesses of the proposed models were discussed.

Acknowledgements We are grateful to Centor and to the industry representative Glen Pacholke for bringing this problem to MISG 2015 and for his valuable input. We are particularly grateful to Centor for performing experiments during the week of the Study Group and for hosting visits from Study Group participants, and to QUT for providing Kinects for the experimental work. We also acknowledge and thank the other team members who worked on this problem: Andrea Babylon, Andrew Cramer, Megan Farquhar, Troy Farrell, Emma Greenbank, Patrick Hassard, Matt Hayes, Jim Hill, Stuart Johnston, Ravi Pethiyagoda, Sarthok Sircar, Kyle Talbot.

\section{References}

[1] Standards Australia. AS/NZS1170.2-Structural design actions part 2: Wind actions, 2011. M64

[2] F. Avanzini and R. Maronga. A modular physically based approach to the sound synthesis of membrane percussion instruments. IEEE Transactions on Audio, Speech, and Language Processing, 18(4):891-902, 2010. doi:10.1109/TASL.2009.2036903 M80, M92, M99 
[3] B. Bank. Energy-based synthesis of tension modulation in strings. In Proceedings of the 12th International Conference on Digital Audio Effects (DAFx-09), 2009. http:

//dafx09.como.polimi.it/proceedings/papers/paper_76.pdf M80, M92

[4] Bert Blocken. 50 years of computational wind engineering: Past, present and future. Journal of Wind Engineering and Industrial Aerodynamics, 129(0):69-102, 2014. doi:10.1016/j.jweia.2014.03.008 M63

[5] Demetres Briassoulis, Antonis Mistriotis, and Anastasios Giannoulis. Wind forces on porous elevated panels. Journal of Wind Engineering and Industrial Aerodynamics, 98(12):919-928, 2010. doi:10.1016/j.jweia.2010.09.006 M63

[6] John Cheung and William Melbourne. Wind loading on porous cladding. In 9th Australasian fluid mechanics conference, 1986.

http://people.eng.unimelb.edu.au/imarusic/proceedings/9/ CheungMelbourne.pdf M64

[7] John Holmes. Wind Loading of Structures. Spon Press, 2001. M63

[8] P. D. Howell, G. Kozyreff, and J. R. Ockendon. Applied Solid Mechanics. Cambridge University Press, 2009. M78

[9] C.W Letchford. Wind loads on rectangular signboards and hoardings. Journal of Wind Engineering and Industrial Aerodynamics, 89(2):135-151, 2001. doi:10.1016/S0167-6105(00)00068-4 M63

[10] Wojciech Okrasiński and Łukasz Płociniczak. A nonlinear mathematical model of the corneal shape. Nonlinear Analysis: Real World Applications, 13(3):1498-1505, 2012. doi:10.1016/j.nonrwa.2011.11.014 M80, M99

[11] D. W. Oplinger. Frequency response of a nonlienar stretched string. Journal of the Acoustical Society of America, 32(12):1529-1538, 1960. doi:10.1121/1.1907948 M80 
[12] Xavier Provot. Deformation constraints in a mass-spring model to describe rigid cloth behaviour. In Graphics interface, pages 147-147. Canadian Information Processing Society, 1995. http://kucg.korea. ac.kr/education/2005/CSCE352/paper/provot95.pdf M67, M68, M69

[13] P.J Richards and M Robinson. Wind loads on porous structures. Journal of Wind Engineering and Industrial Aerodynamics, 83(13):455-465, 1999. doi:10.1016/S0167-6105(99)00093-8 M63

[14] A.P. Robertson, Ph. Roux, J. Gratraud, G. Scarascia, S. Castellano, M. Dufresne de Virel, and P. Palier. Wind pressures on permeably and impermeably-clad structures. Journal of Wind Engineering and Industrial Aerodynamics, 90(45):461-474, 2002. Bluff Body Aerodynamics and Applications. doi:10.1016/S0167-6105(01)00210-0 M63, M64, M66, M106

[15] T. Tolonen, V. Välimäki, and M. Karjalainen. Modeling of tension modulation nonlinearity in plucked strings. IEEE Transactions on Speech and Audio Processing, 8(3):300-310, 2000. doi:10.1109/89.841212 M80, M92

\section{Author addresses}

1. Cameron L. Hall, Mathematical Institute, University of Oxford, Woodstock Road, Oxford OX2 6GG, United KingDOM. mailto:hall@maths.ox.ac.uk

2. Matthew S. Mason, School of Civil Engineering, University of Queensland, Brisbane, Qld 4072, Australia. mailto:matthew. mason@uq.edu. au

3. Steven Psaltis, School of Mathematical Sciences, Queensland University of Technology, Brisbane, Qld 4000, Australia. 
mailto:steven.psaltis@qut.edu.au

4. Matthew Chan, School of Mathematics and Statistics, University of Sydney, Sydney, NSW 2006, Australia.

mailto:matthew. chan@sydney . edu . au

5. Eamon Conway, School of Mathematical Sciences, Queensland University of Technology, Brisbane, Qld 4000, Australia. mailto:e1. conway@qut.edu.au

6. Brody Foy, School of Mathematical Sciences, Queensland University of Technology, Brisbane, Qld 4000, Australia.

mailto:b.foy@qut.edu.au

7. Sayyed R. Mirnaziry, School of Mathematical and Physical Sciences, University of Technology Sydney, Ultimo, NSW 2007, Australia. mailto:sayyed.r.mirnaziry@student .uts.edu .au

8. Danya Rose, School of Mathematics and Statistics, University of Sydney, Sydney, NSW 2006, Australia.

mailto:danya.rose@sydney.edu.au

9. Stephen Taylor, Mathematics Department, University of Auckland, Auckland, Private Bag 92019, NEw ZEALAnd.

mailto:s.taylor@auckland.ac.nz

10. Jakub Tomczyk, School of Mathematics and Statistics, University of Sydney, Sydney, NSW 2006, Australia.

mailto: jakub. tomczyk@maths. usyd.edu.au 